\title{
The Jules Horowitz Reactor Research Project: A New High Performance Material Testing Reactor Working as an International User Facility - First Developments to Address R\&D on Material
}

\author{
Gilles BIGNAN ${ }^{1}$, Christian COLIN ${ }^{1}$, Jocelyn PIERRE ${ }^{1}$, Christophe BLANDIN ${ }^{1}$, \\ Christian GONNIER ${ }^{1}$, Michel AUCLAIR ${ }^{2}$, Franck ROZENBLUM ${ }^{2}$ \\ ${ }^{1}$ CEA-DEN-DER, JHR Project (Cadarache, France) \\ ${ }^{2}$ CEA-DEN-DRSN, Service d'Irradiations en Réacteurs et d'Etudes Nucléaires, SIREN (Saclay, France)
}

The Jules Horowitz Reactor (JHR) is a new Material Testing Reactor (MTR) currently under construction at CEA Cadarache research center in the south of France. It will represent a major research infrastructure for scientific studies dealing with material and fuel behavior under irradiation (and is consequently identified for this purpose within various European road maps and forums; ESFRI, SNETP...). The reactor will also contribute to medical Isotope production.

The reactor will perform R\&D programs for the optimization of the present generation of Nuclear Power Plans (NPPs), will support the development of the next generation of NPPs (mainly LWRs) and also will offer irradiation capabilities for future reactor materials and fuels.

JHR is fully optimized for testing material and fuel under irradiation, in normal, incidental and accidental situations:

- with modern irradiation loops producing the operational condition of the different power reactor technologies ;

- with major innovative embarked in-pile instrumentation and out-pile analysis to perform highquality R\&D experiments ;

- with high thermal and fast neutron flux capacity and high dpa rate to address existing and future NPP needs.

JHR is funded and steered and will be operate as an international user-facility open to international collaboration. This lead to the following topics:

- the existence of an international consortium gathering the funding organizations to steer the project ;

- the setting-up of an international scientific community around JHR through seminars, working groups to optimize the experimental capacity versus future $R \& D$ needs ;

- the preparation of the first JHR International Program potentially open to non-members of the JHR consortium.

It will answer needs expressed by the scientific community (R\&D institutes, TSO...) and the industrial companies (utilities, fuel vendors...). Consequently, the JHR facility will become a major scientific hub for cutting edge research and material investigations (multilateral support to complete cost effective studies avoiding fragmentation of scientific effort, access to developing countries to such state of the art research reactor facilities, supra national approach....).

Considering material behavior under irradiation, such studies is most of the time associated with a complex multi-physical modelling of the materials' behaviors. It requires well controlled and instrumented irradiation experiments in material testing reactors.

This paper gives an up-to-date status of the construction (Fig. 1) and of the developments performed to build the future experimental capacity dedicated to the material irradiations in JHR reactor. In-core and in reflector devices will be presented (Fig. 2), corresponding to large ranges of temperature and neutrons flux for the irradiation conditions. A special attention focuses on the improvement of the thermal stability and gradients of the interest zones in samples despite strong gamma heating and on 
an improvement of the instrumentation devoted to the experiments. Some specific devices in support of LWR type reactors will be described such as equipment designed for the qualification of Reactor Pressure Vessel (RPV) steels, for the study of the stress corrosion cracking assisted by irradiation phenomena (IASCC), or for the studies of creep-swelling of structural materials.

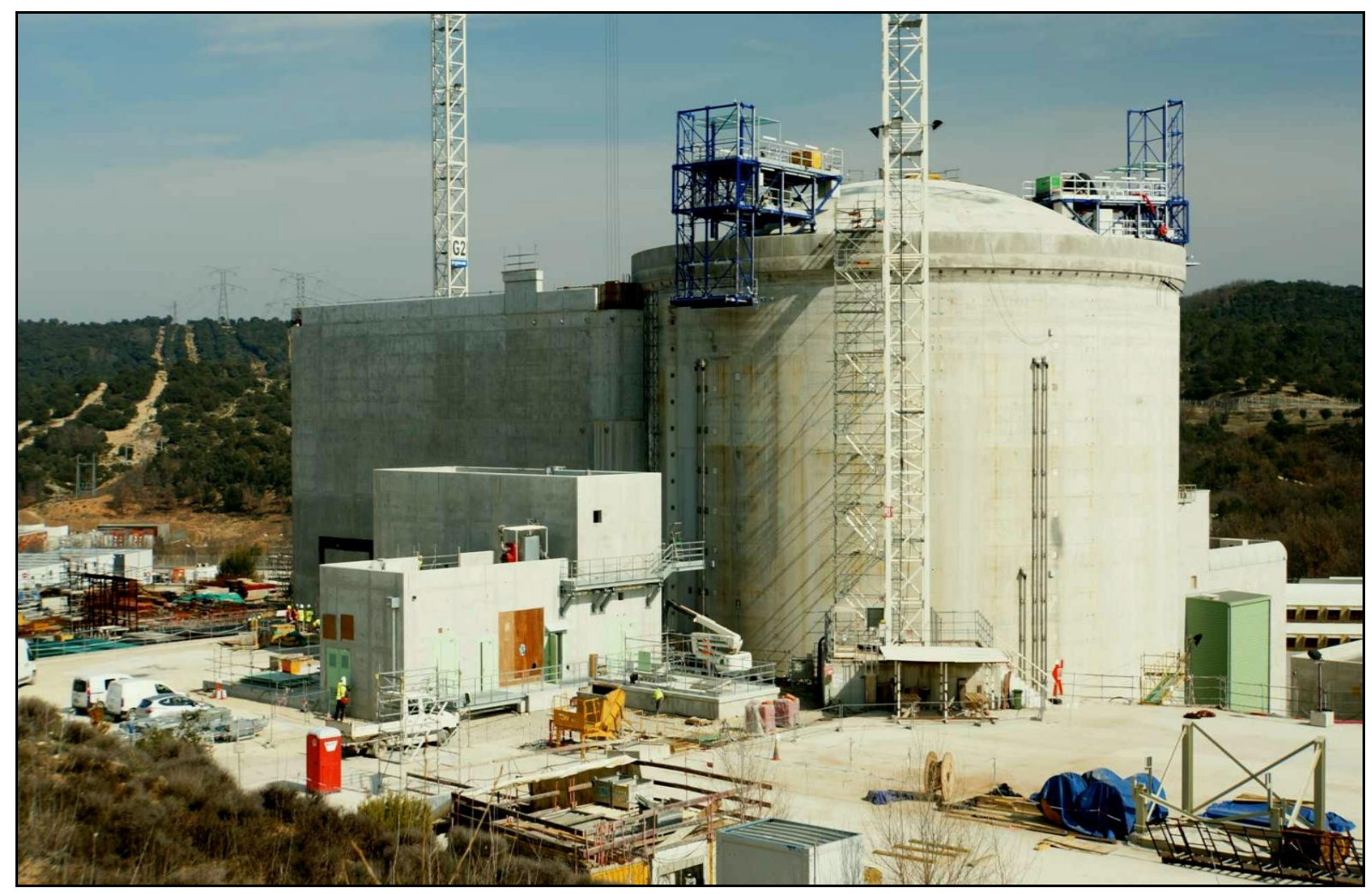

Fig. 1: General view of the JHR building (September 2015).

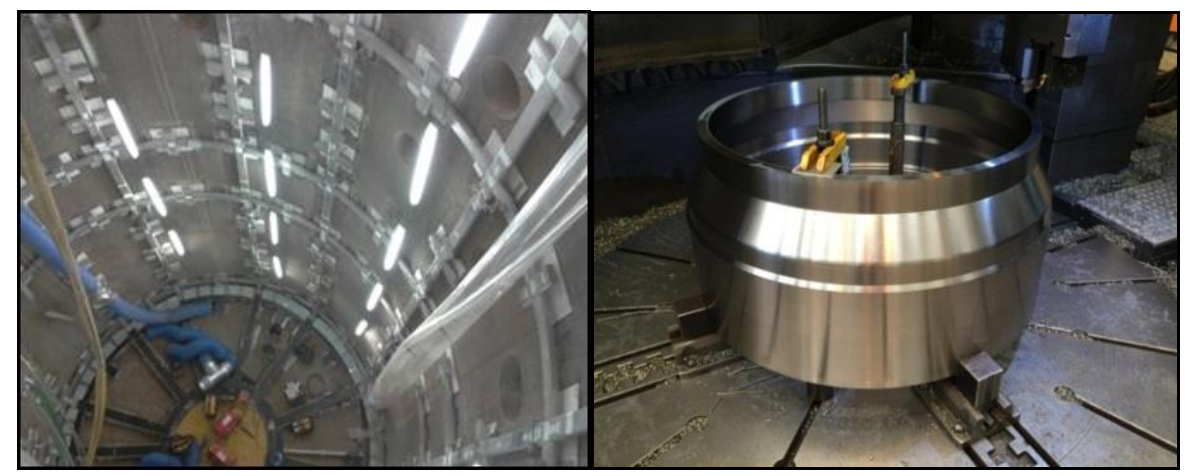

Fig. 2: reactor pool (left) and part a reactor block (right) of the JHR (September 2015).

\section{References}

[1] G. Bignan, X. Bravo, "The Jules Horowitz Reactor: A new high performance MTR (Material Testing Reactor) working as an International User Facility in support to Nuclear Industry, Public Bodies and Research Institutes", Nuclear Energy International Journal, December 2014, pp. 26-30. 
JHR: a future Reference International User Facility

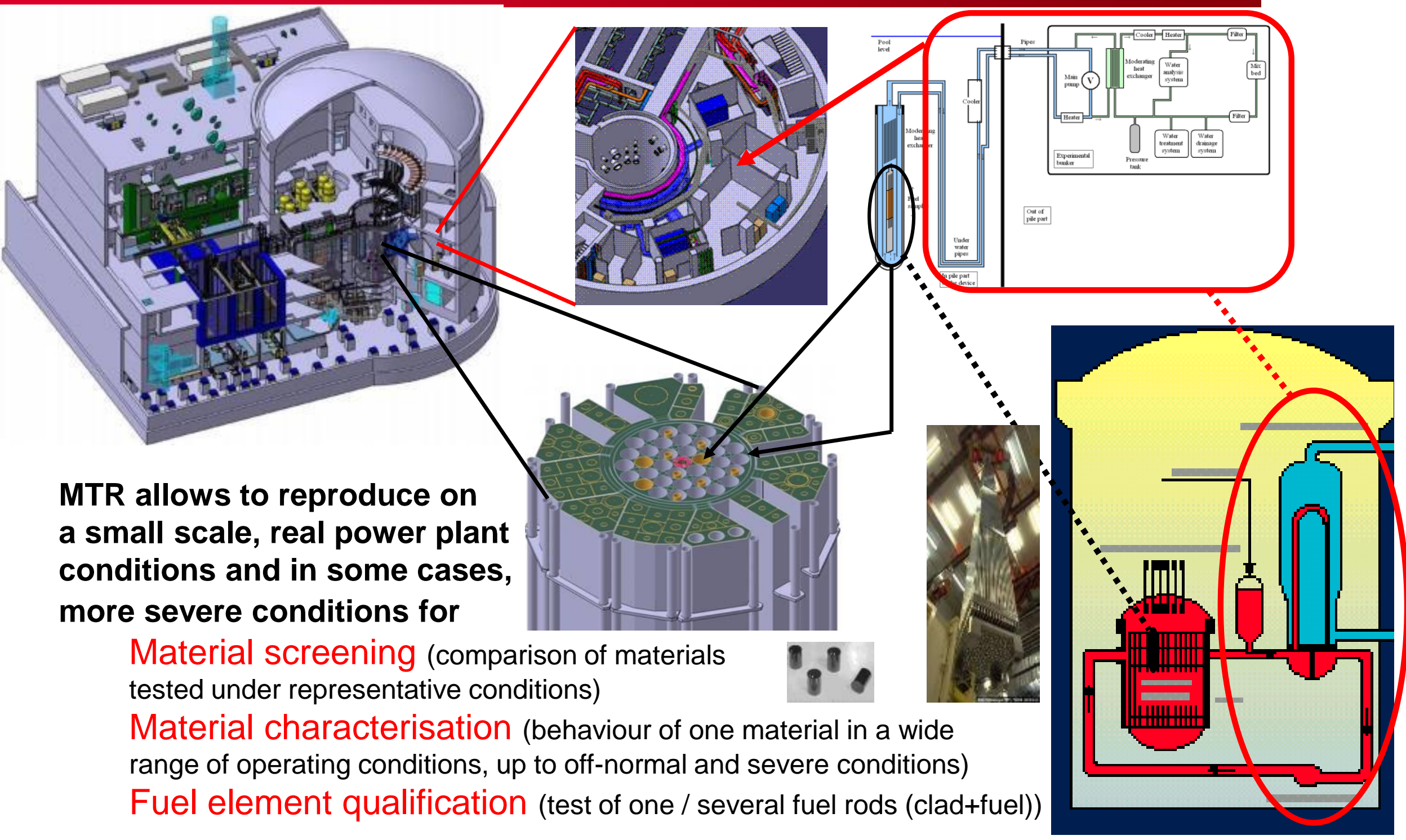




\section{An Ageing fleet of MTR in Europe}

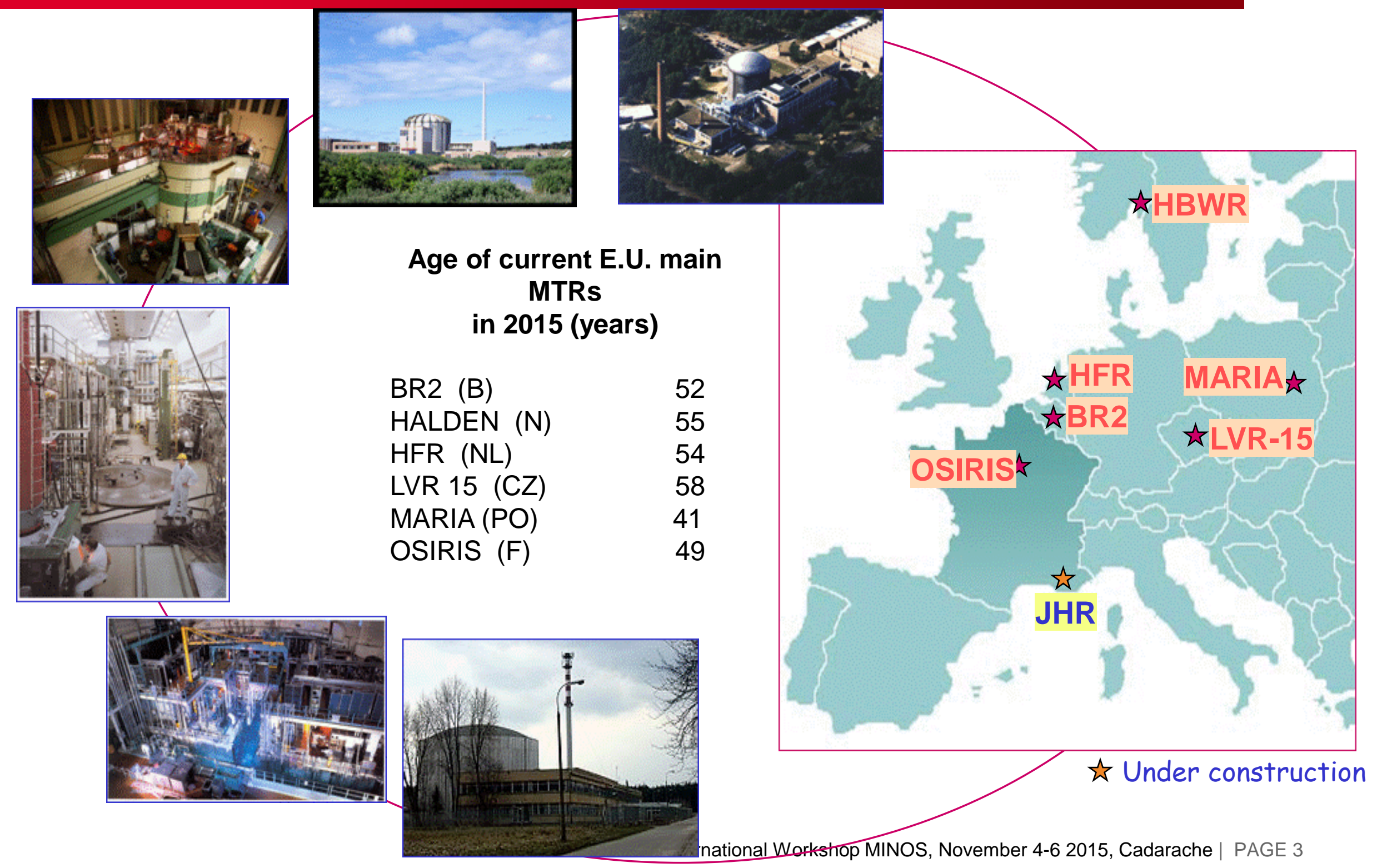


1) $R \& D$ in support to nuclear Industry

- Safety and Plant life time management (ageing \& new plants) Fuel behavior validation in incidental and accidental situation Assess innovations and related safety for future NPPs

2) Radio-isotopes supply for medical application

\section{MOLI production}

JHR will supply $25 \%$ of the European demand (today about 8 millions protocols/year) and up to $50 \%$ upon specific request
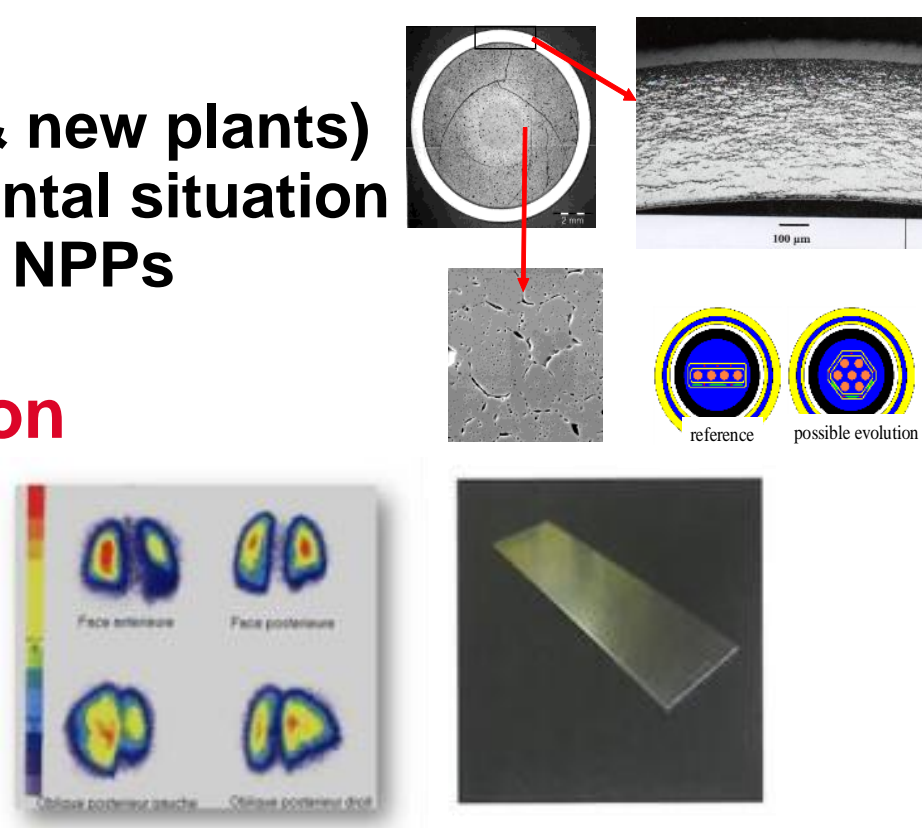

3) A key tool to support expertise

Training of new generations (JHR simulator, secondee's program) Maintaining a national expertise staff and credibility for public acceptance Assessing safety requirements evolution and international regulation harmonisation

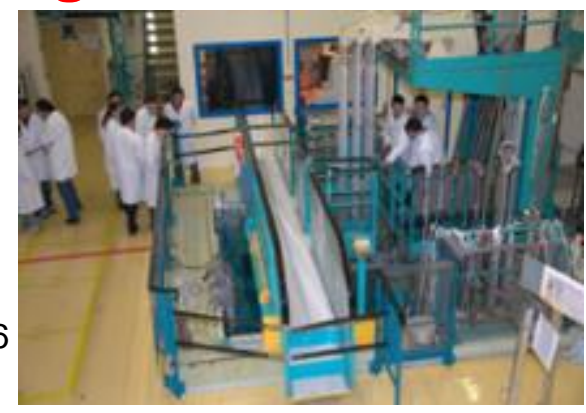




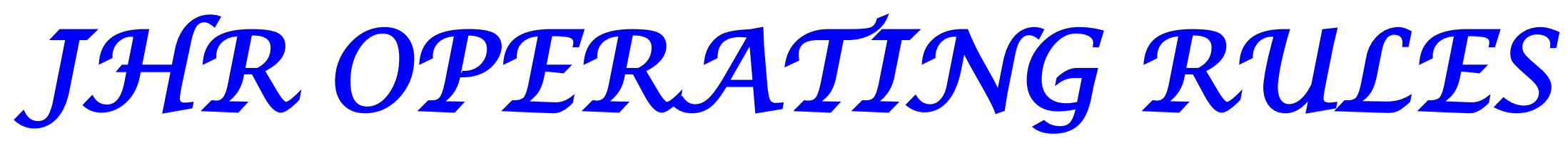




\section{9/03/2007 Signature of the JHR consortium}

JHR consortium gathers organizations which take part financially in the construction of JHR (1 representative / organization)

JHR Consortium current partnership: Research centers \& Industrial companies

\section{Studsvik}
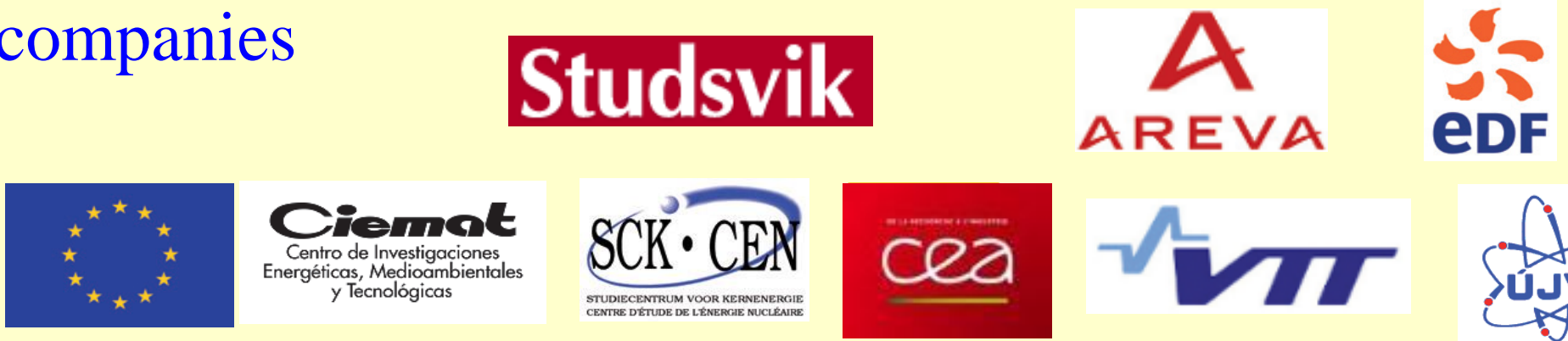
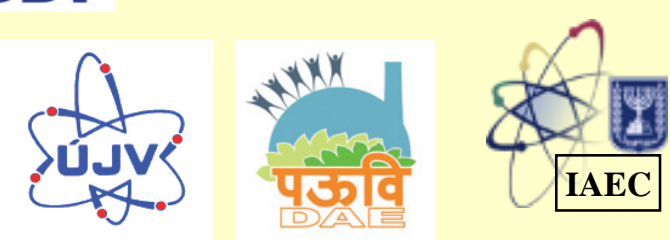

Associated Partnership(JAEA))

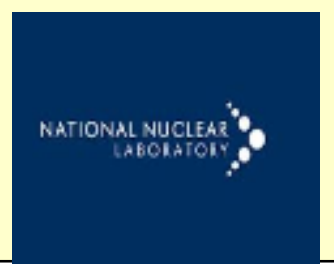

In some cases, the organization (member of the JHR consortium) is itself the representative of a national domestic consortium which gathers organizations among industry, academics, R\&D organizations, TSO, or Safety Authority 
Project leader appointment

Validation of operation plan, business

strategy, economy of the project

Nuclear safety; Technical and

Economical performance (operation cost)

Operation plan fulfilment programs definition (preparation of next

Operation Plan with users)
Governing Board

(JHR Consortium Members)

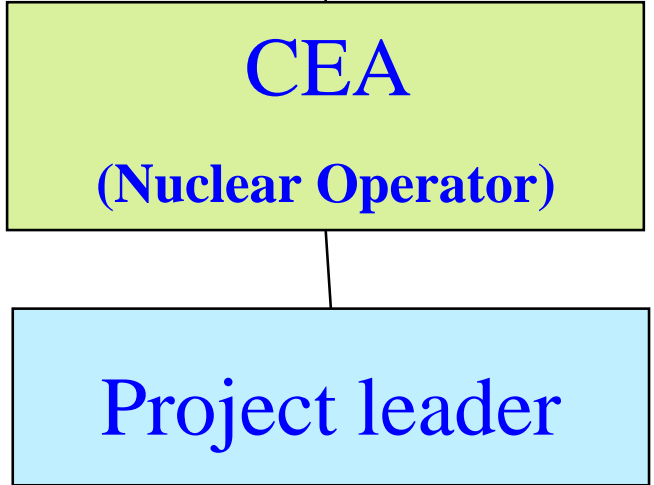

JHR Reference Operation Plan (4 years plan)

For Members of the Consortium and Non-Members

\section{Proprietary Programs}

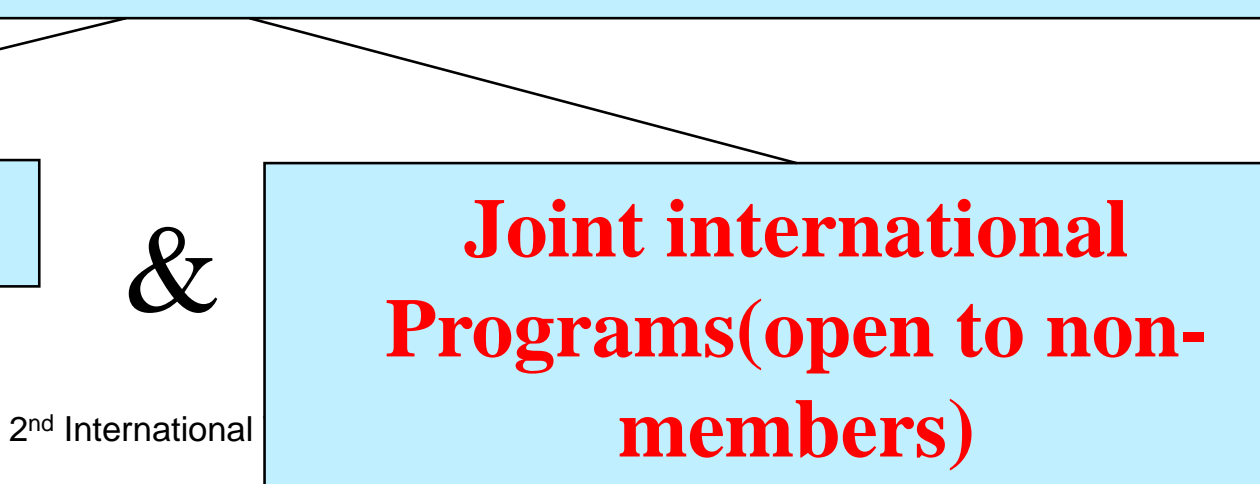




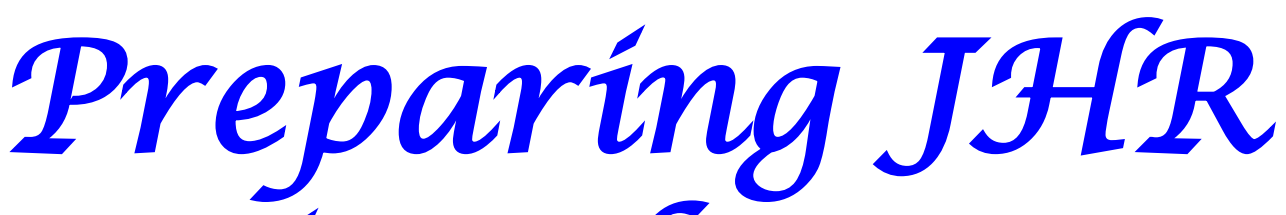

International Community:

- The yearly seminar

- The 3 Working Groups

- The Secondee Program

- The recent ICERR

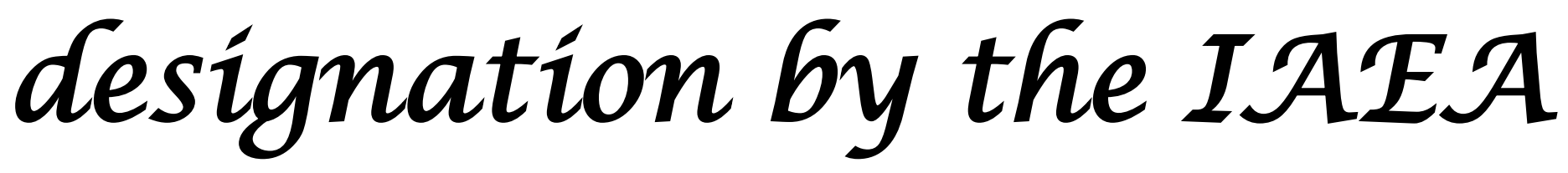




\section{Create international scientific networks}

Make available facilities and experience of mature R\&D centres in the field of peaceful uses of Nuclear Energy to affiliates

Lead innovative joint programs with shared results

Host international scientists / engineers

Provide "hands on" nuclear education "in the field"

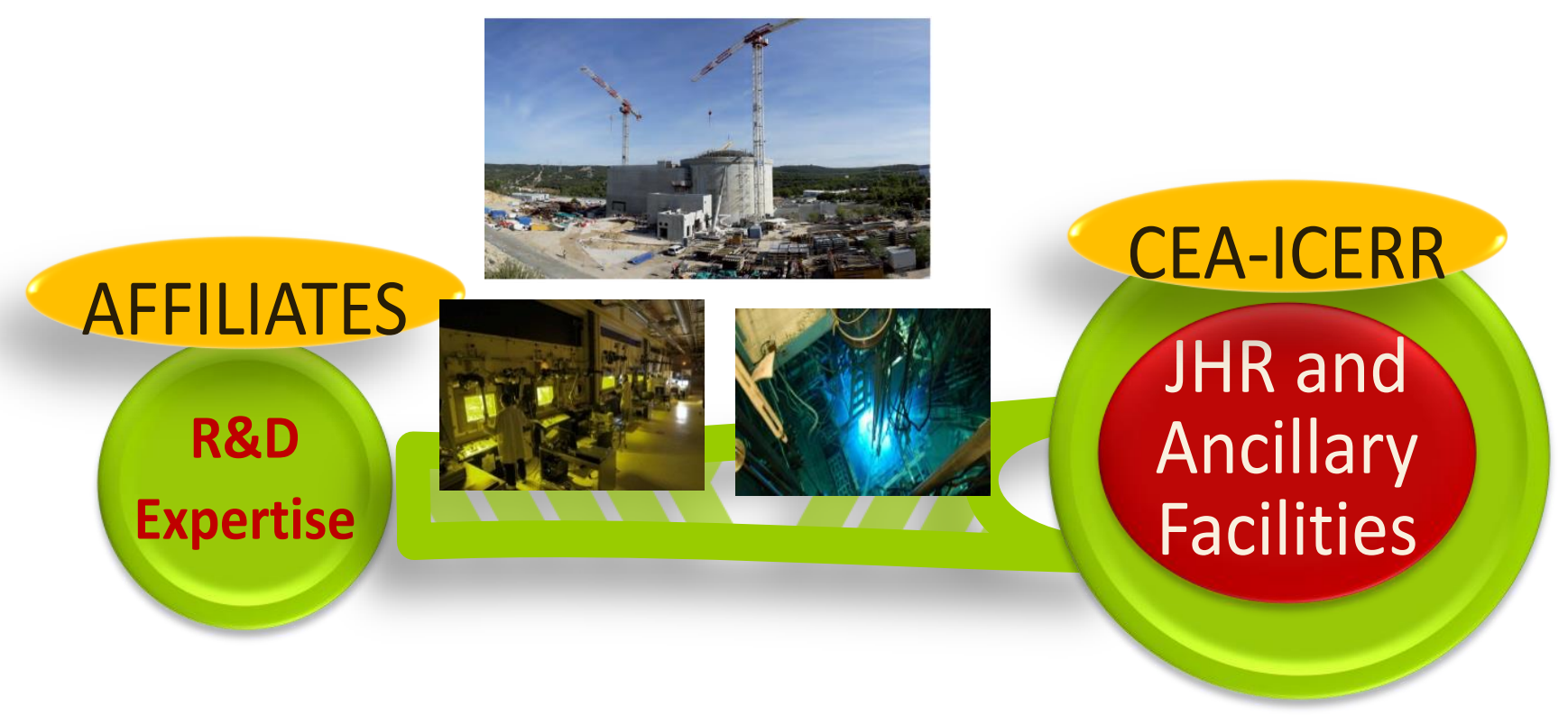


LECI : Hot Lab on

Materials

- Hands-On Training

(Equipments)

- R\&D Projects

ORPHEE : Neutron beams

- Hands-On Training (Equipments)

- R\&D Projects

ZEPHYR : LPR

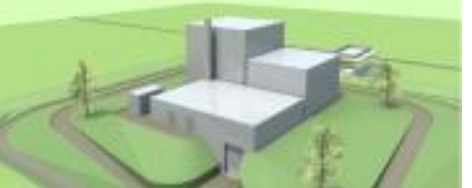

New Projects

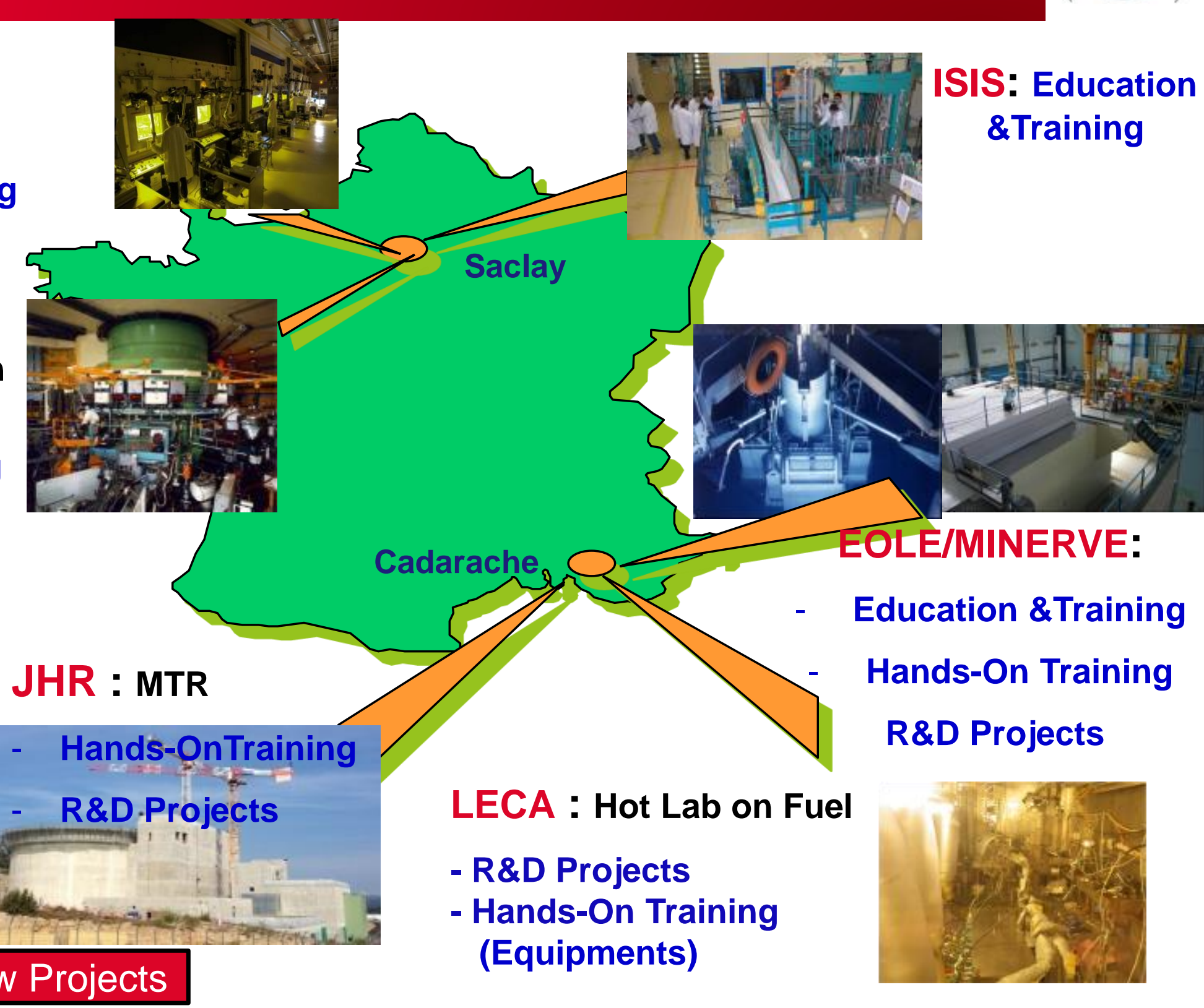




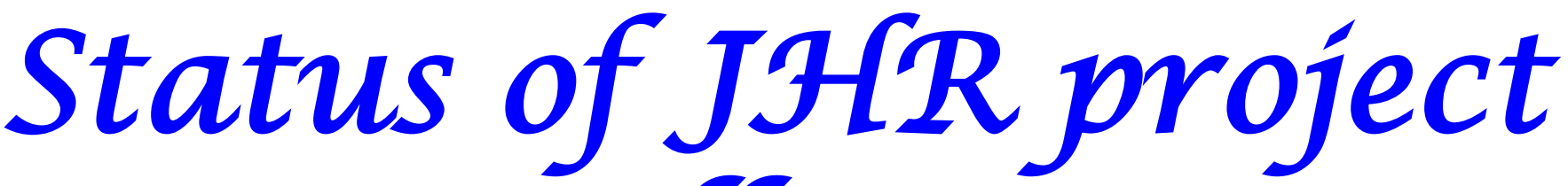 Fall 2015}




\section{Reactor building}
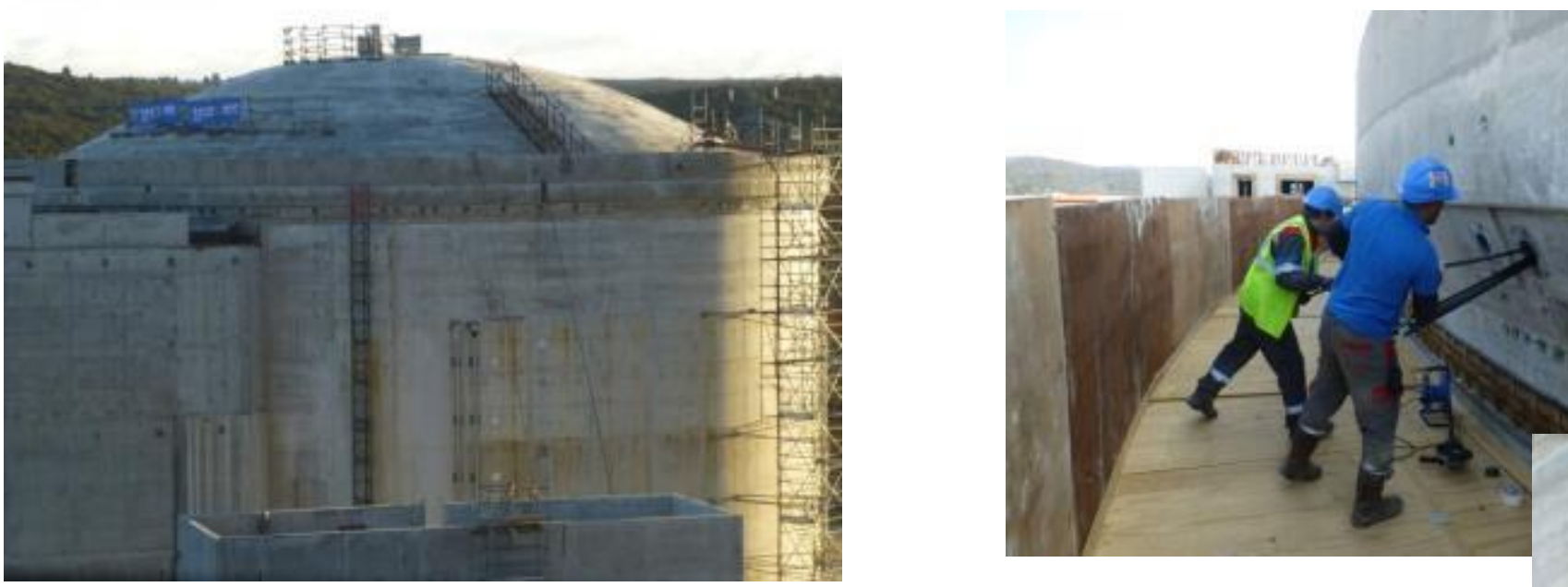

Removal of dôme formwork

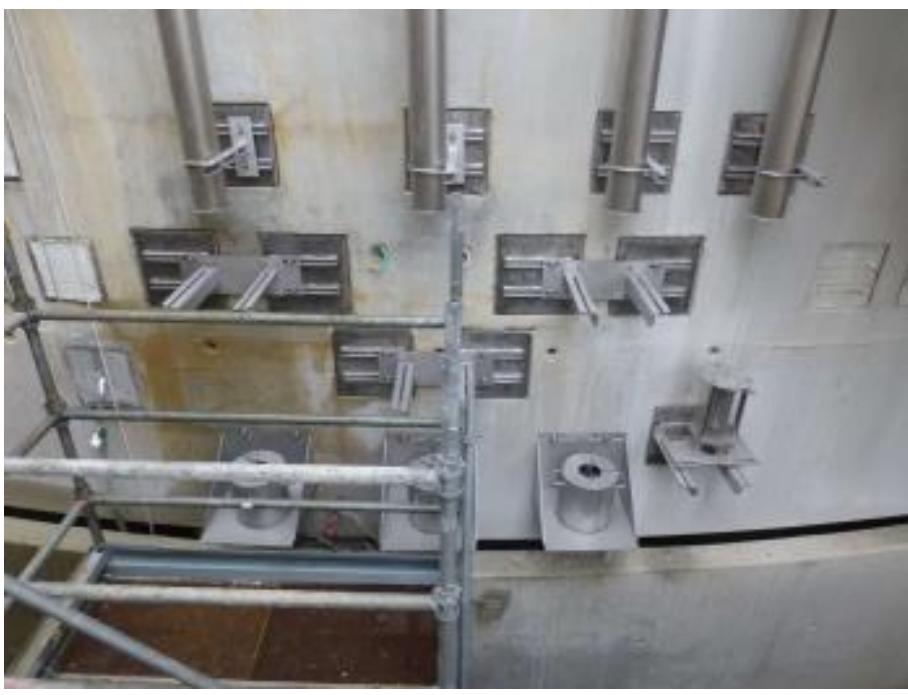

Installation of prestressing cables

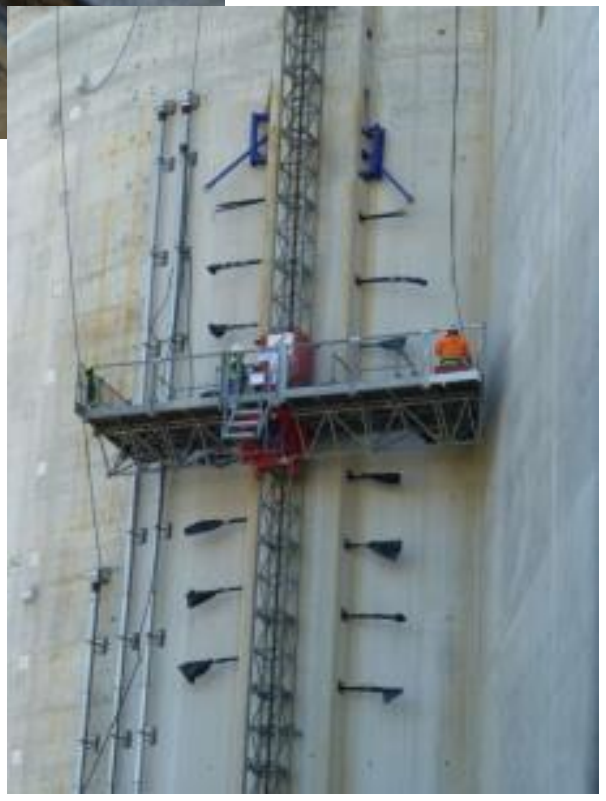

Installation of specific devices for reactor containment monitoring 


\section{Beginning of components manufacturing}

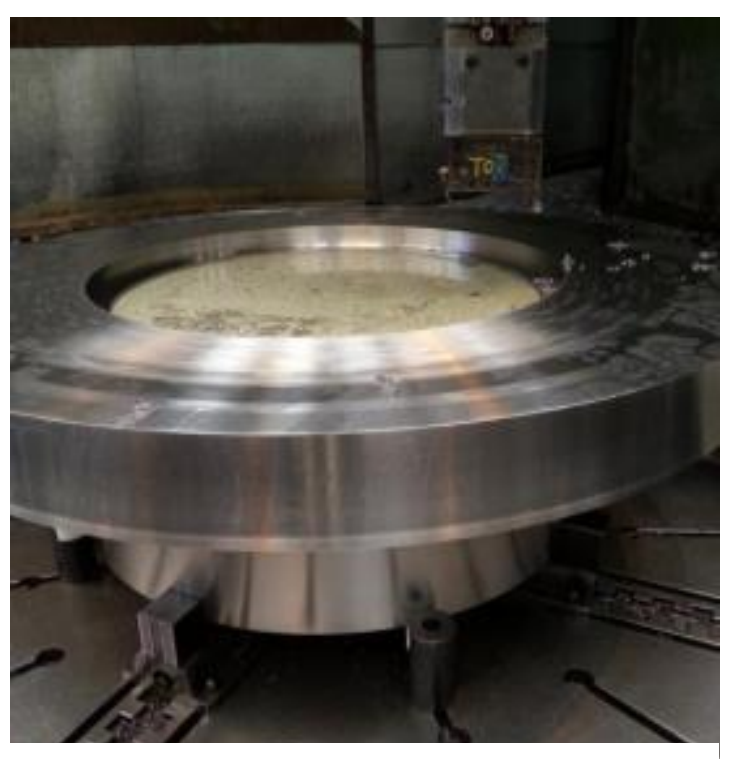

Machining of bottom plug flange (S12/2015)

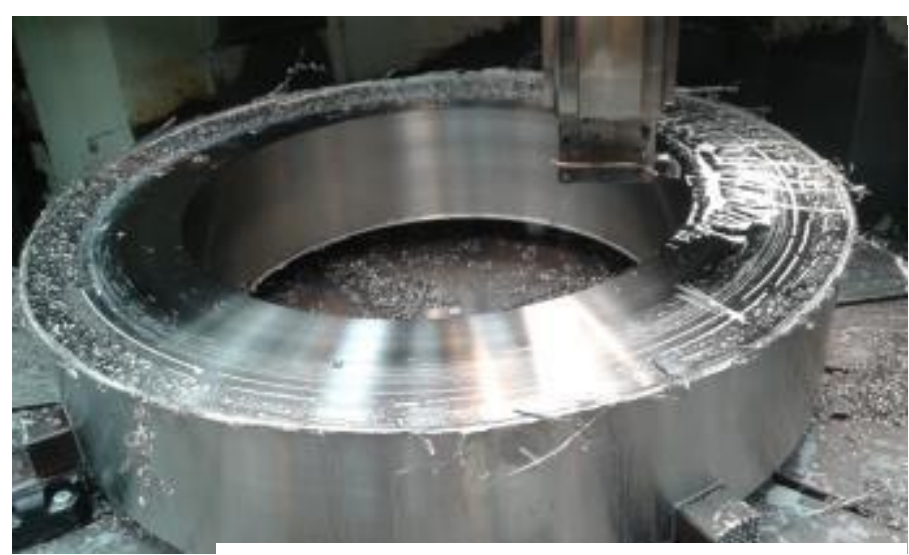

Water Box REP (S18/2015)

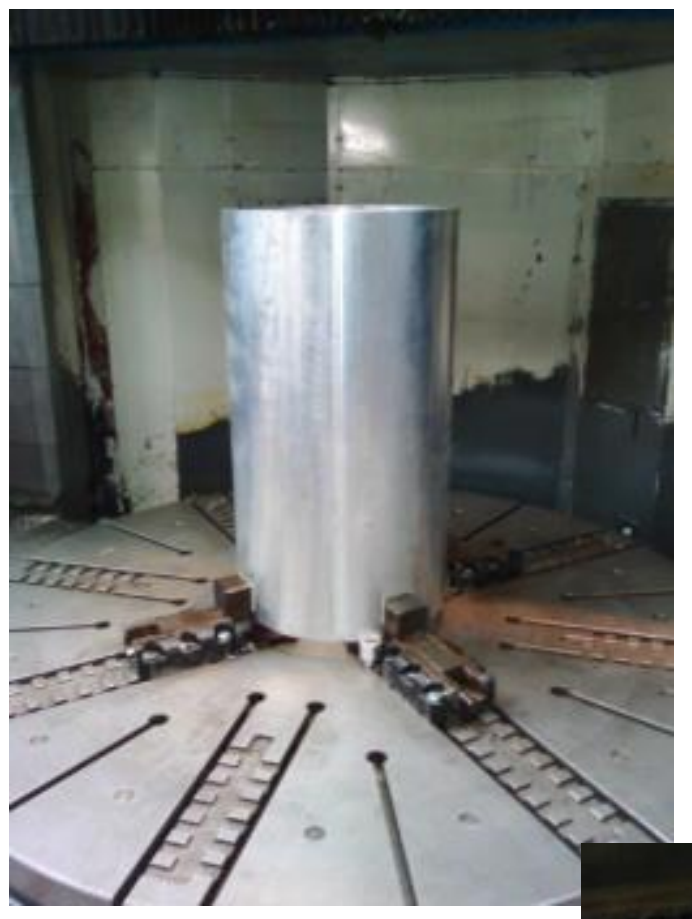

Main vessel

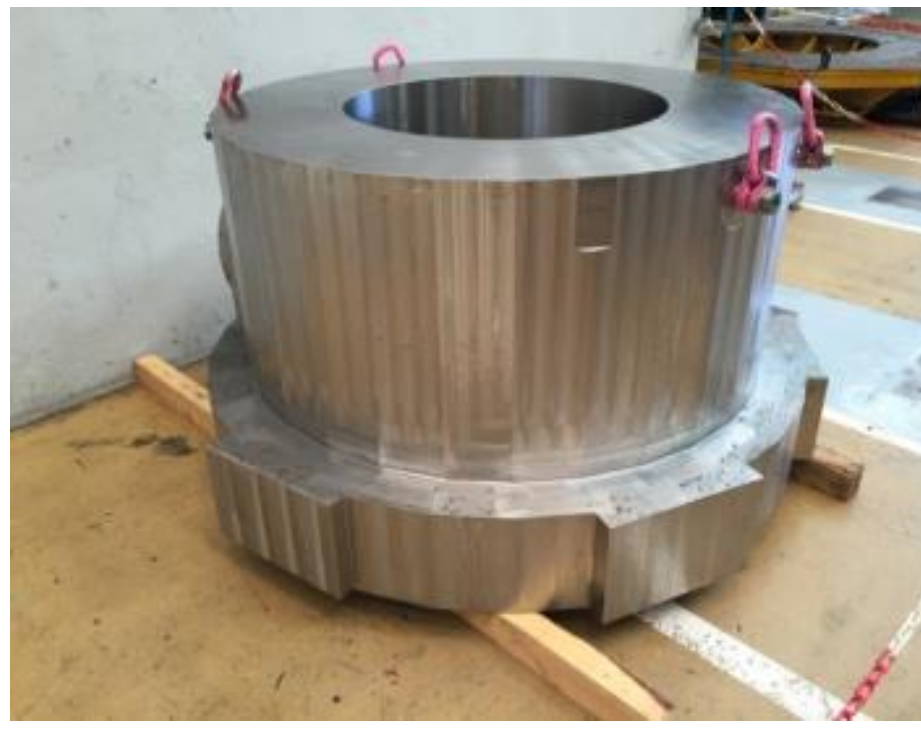

Water Box RPP (S12/2015)

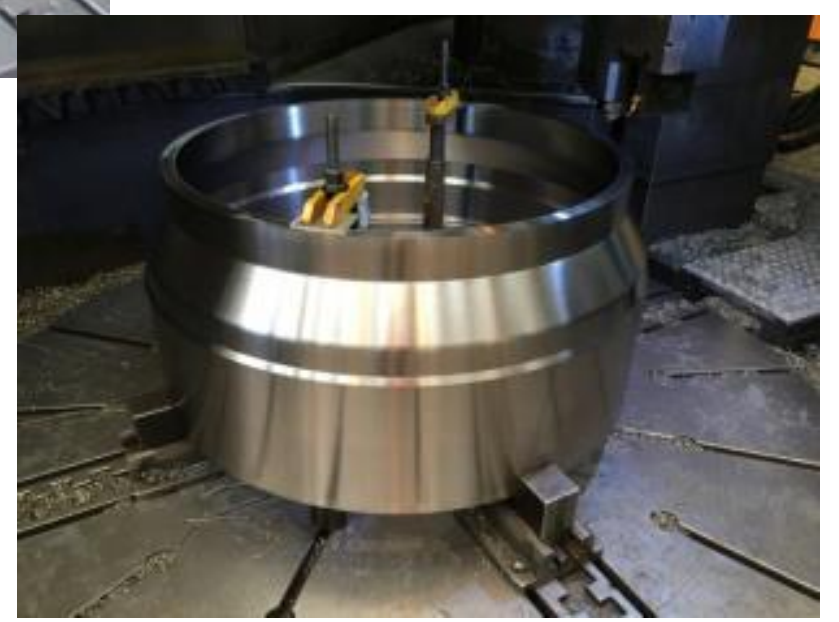

Bottom plug shell (S18/2015) 
$J \mathcal{H R}$ design and performances

First fleet of experimental Devices under development For Material Investigation 
A facility dedicated to experimental purposes :

- A modern facility :

- Large experimental areas
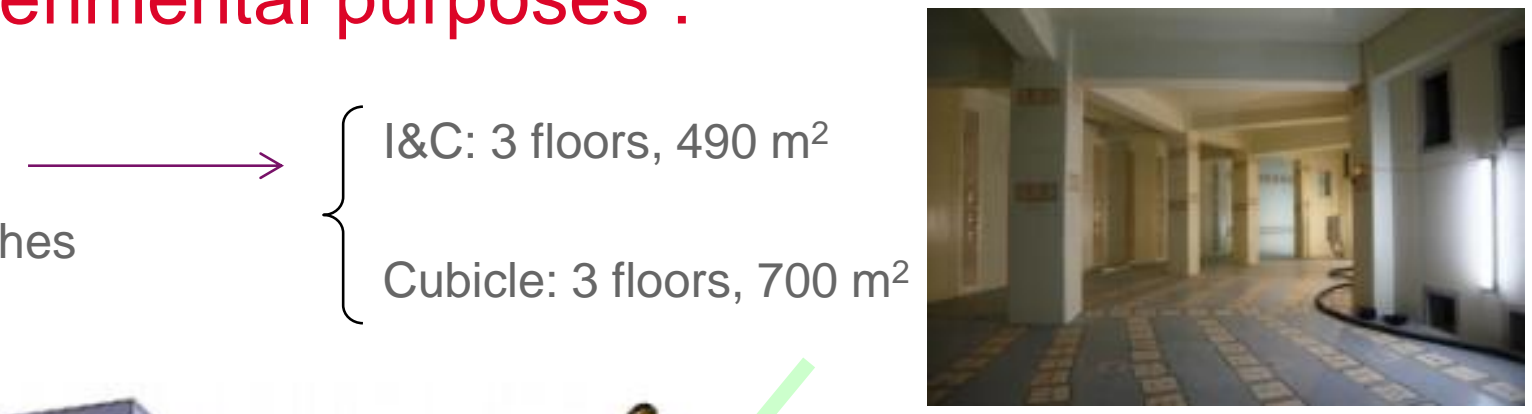

- Fission Product Laboratory

- Chemistry Laboratory...
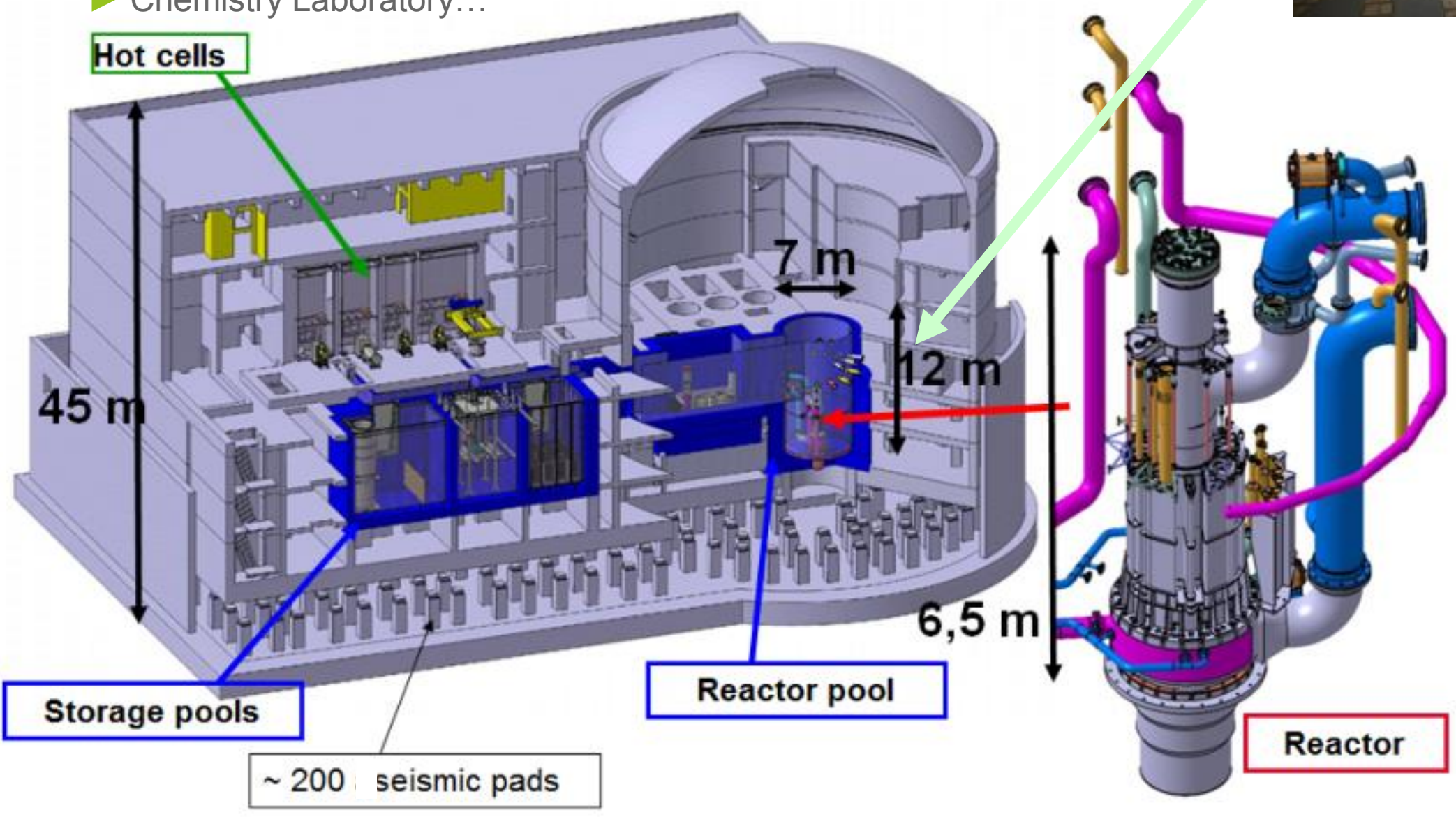


\section{JHR facility \& experimental capacity: First fleet of irradiation material devices}

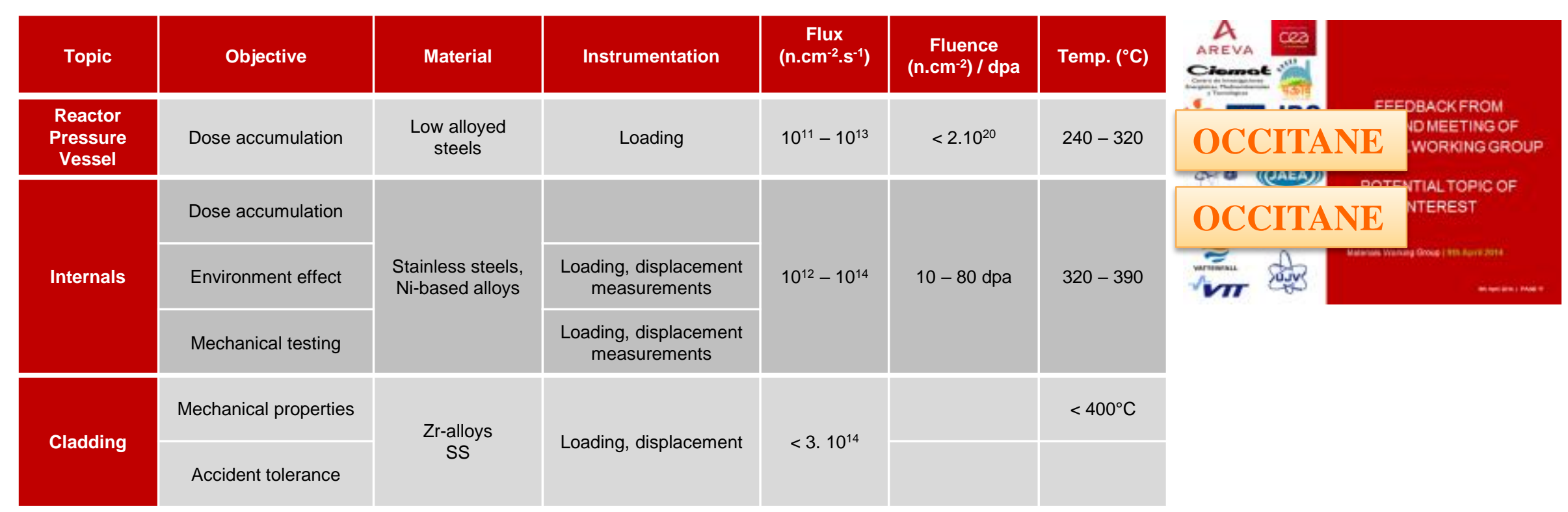

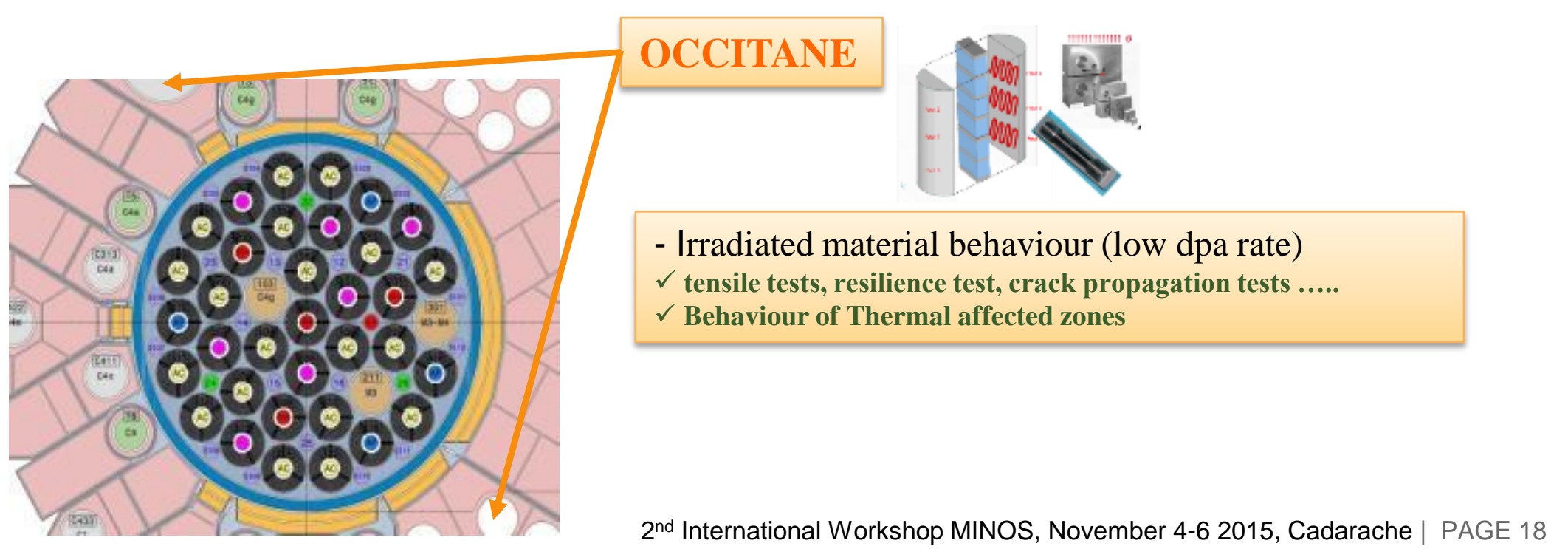



First fleet of irradiation material devices

\begin{tabular}{|c|c|c|c|c|c|c|c|}
\hline Topic & Objective & Material & Instrumentation & $\begin{array}{c}\text { Flux } \\
(\text { n.cm } \\
\left.-2 \cdot \mathrm{s}^{-1}\right)\end{array}$ & $\begin{array}{c}\text { Fluence } \\
\left(\mathrm{n} . \mathrm{cm}^{-2}\right) / \mathrm{dpa}\end{array}$ & Temp. $\left({ }^{\circ} \mathbf{C}\right)$ & \\
\hline $\begin{array}{c}\text { Reactor } \\
\text { Pressure } \\
\text { Vessel }\end{array}$ & Dose accumulation & $\begin{array}{l}\text { Low alloyed } \\
\text { steels }\end{array}$ & Loading & $10^{11}-10^{13}$ & $<2.10^{20}$ & $240-320$ & \\
\hline \multirow{3}{*}{ Internals } & Dose accumulation & \multirow{3}{*}{$\begin{array}{l}\text { Stainless steels, } \\
\text { Ni-based alloys }\end{array}$} & & \multirow{3}{*}{$10^{12}-10^{14}$} & \multirow{3}{*}{$10-80 \mathrm{dpa}$} & \multirow{3}{*}{$320-390$} & \\
\hline & Environment effect & & $\begin{array}{l}\text { Loading, displacement } \\
\text { measurements }\end{array}$ & & & & \\
\hline & Mechanical testing & & $\begin{array}{l}\text { Loading, displacement } \\
\text { measurements }\end{array}$ & & & & \\
\hline \multirow{2}{*}{ Cladding } & Mechanical properties & \multirow{2}{*}{$\begin{array}{l}\text { Zr-alloys } \\
\text { SS }\end{array}$} & \multirow{2}{*}{ Loading, displacement } & \multirow{2}{*}{$<3.10^{14}$} & & $<400^{\circ} \mathrm{C}$ & IV \\
\hline & Accident tolerance & & & & & & \\
\hline
\end{tabular}

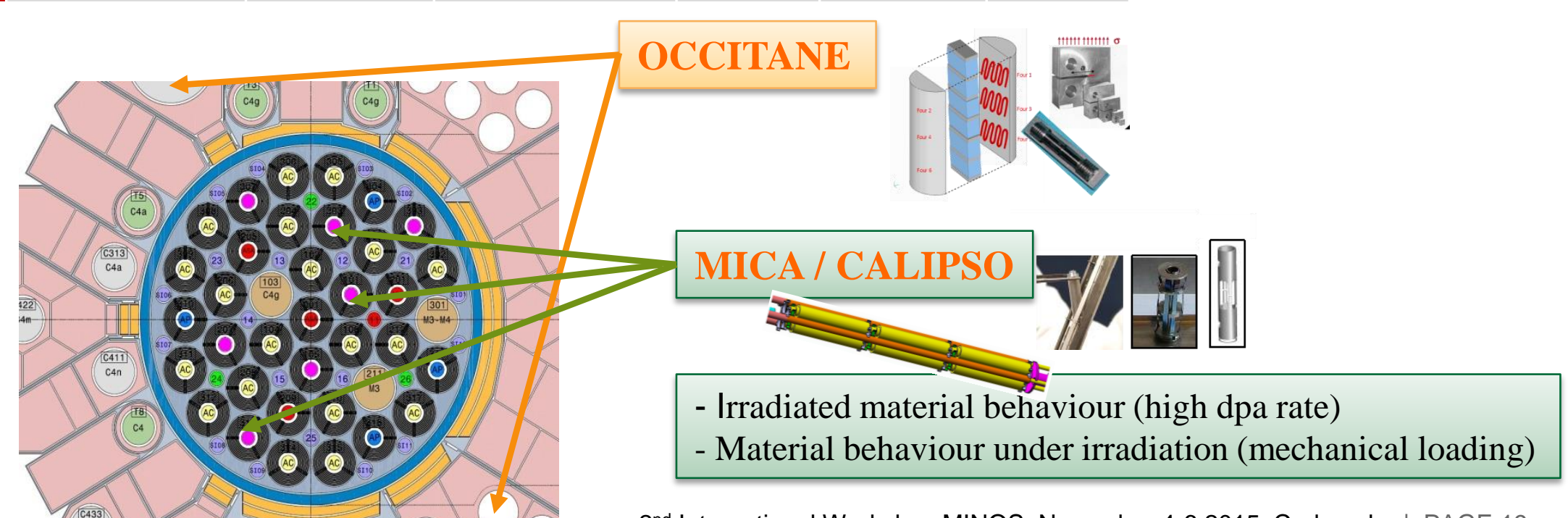


cea den JHR facility \& experimental capacity: First fleet of irradiation material devices

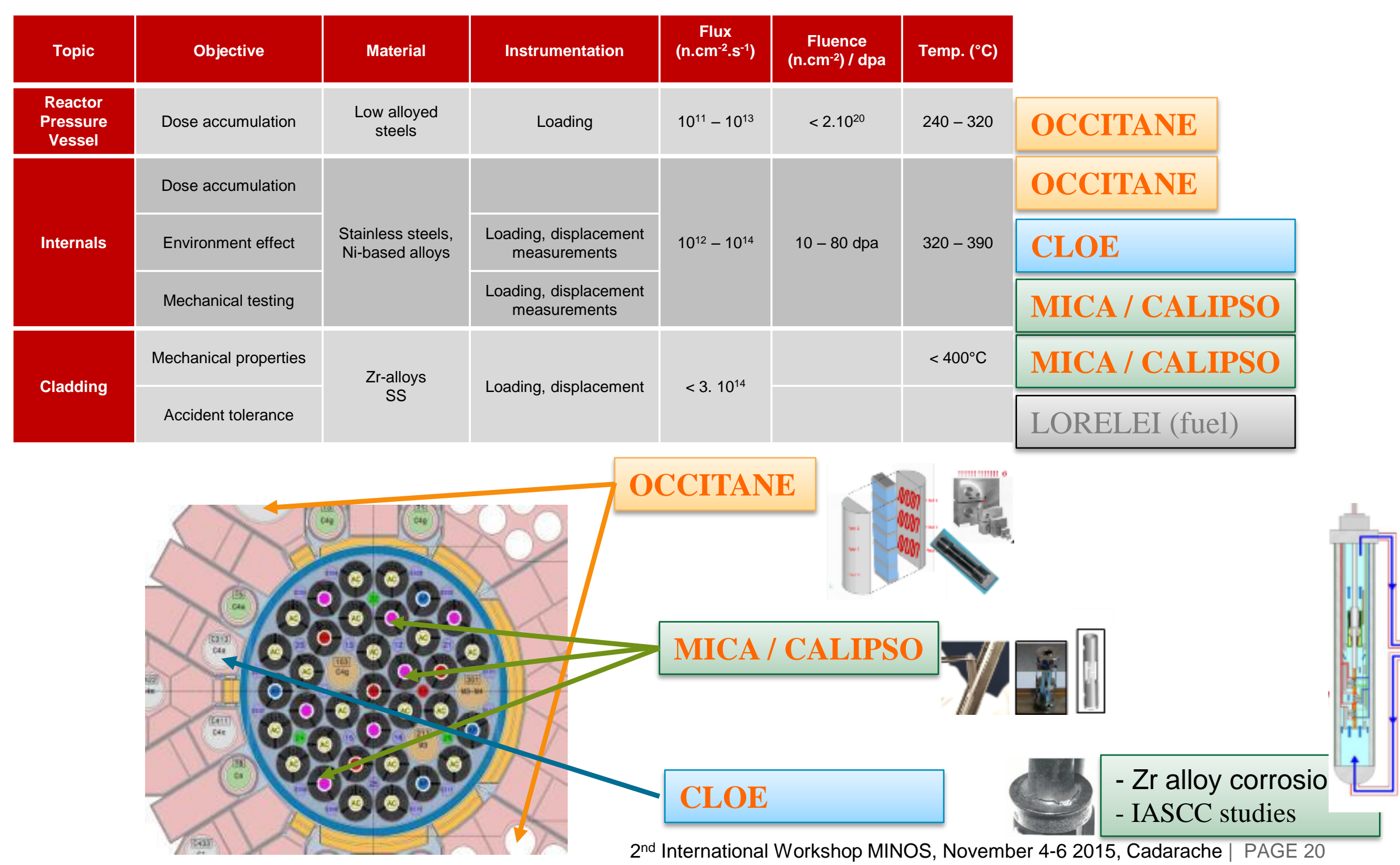




\section{ceaden MICA test device: "Standard" configuration}

MICA : Material Irradiation CApsule

Based on OSIRIS technologies: CHOUCA / PHAETON

$\Rightarrow$ at least, the same performances

Available at JHR start-up

Experimental volume:

Upper volume (instrumentation):

Lower volume (instrumentation):

$\phi 24 \mathrm{~mm} \times 600 \mathrm{~mm}$

$\phi 24 \mathrm{~mm} \times 2400 \mathrm{~mm}$ (max.)

$\phi 24 \mathrm{~mm} \times 700 \mathrm{~mm}$ (max)

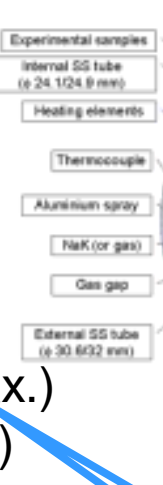

"Standard" MICA

* Static NaK coolant

* $\mathrm{T}<450^{\circ} \mathrm{C}$

* Many samples

* Simplified instrumentation: thermocouples

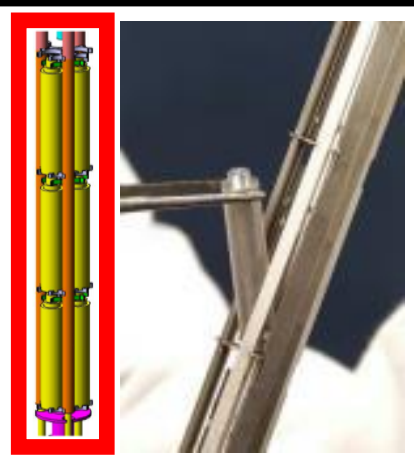

$\rightarrow$ Investigation of physical properties of material

(vs flux, fluence and temperature) under high dpa

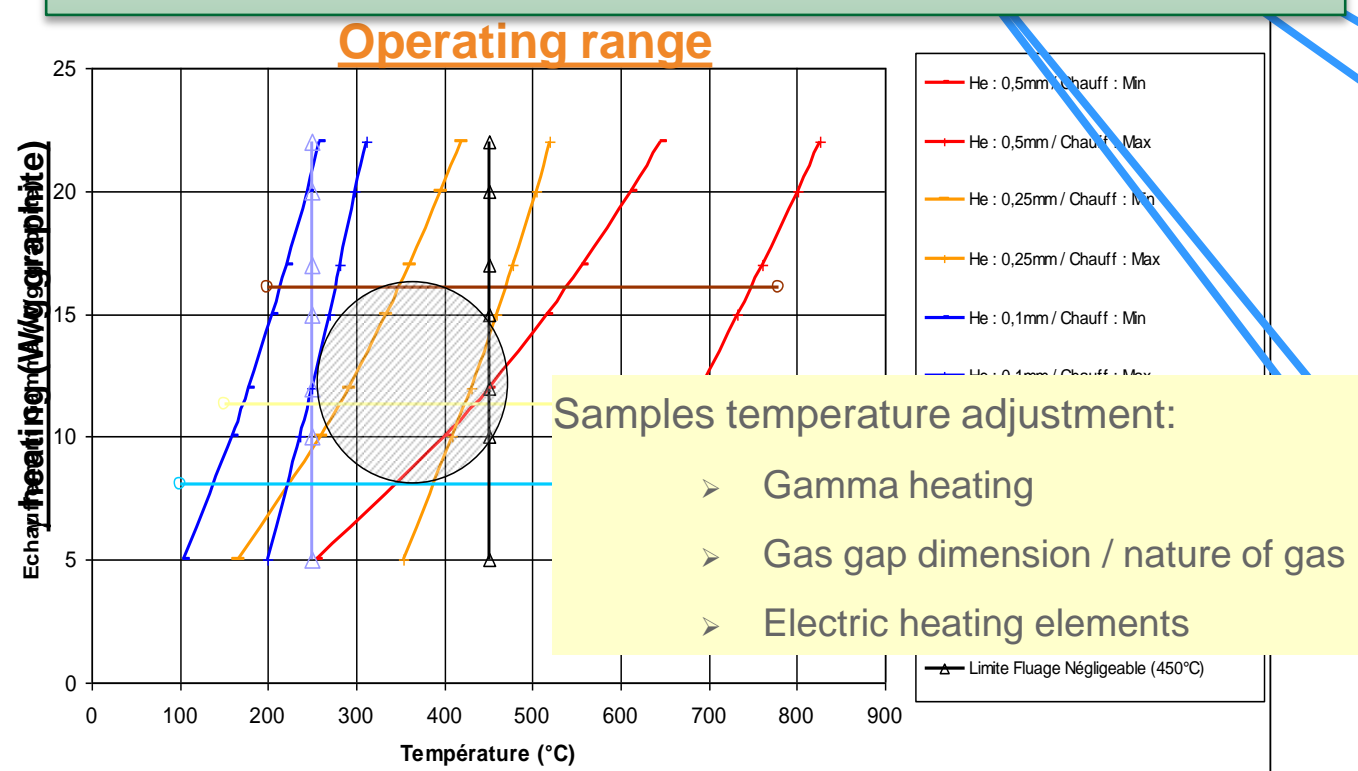

$2^{\text {nd }}$ International Workshop MINOS, November 4-6 2015, Cadarache

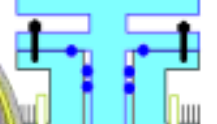

Experimental area \& $\mathrm{NaK}$

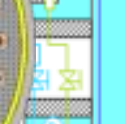

Ш

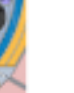




\section{ceaden MICA test device: "HT" configuration}

$\checkmark$ MICA : Material Irradiation CApsule

$\checkmark$ Based on OSIRIS technologies: CHOUCA / PHAETON

$\Rightarrow$ at least, the same performances

$\checkmark$ Available at JHR start-up

$\checkmark$ Experimental volume:

$\phi 24 \mathrm{~mm} \times 600 \mathrm{~mm}$

Upper volume (instrumentation):

$\phi 24 \mathrm{~mm} \times 2400 \mathrm{~mm}$ (max.)

Lower volume (instrumentation):

$\phi 24 \mathrm{~mm} \times 700 \mathrm{~mm}(\max )$

\begin{tabular}{|c|c|c|}
\hline $\begin{array}{l}\text { "Standard" MICA } \\
\text { *tatic NaK coolant } \\
\text { T }<450^{\circ} \mathrm{C} \\
\text { Many samples } \\
\text { Simplified instrumentation: } \\
\text { thermocouples }\end{array}$ & $\begin{array}{l}\text { "Instrumented" MICA } \\
\text { Static NaK coolant } \\
\text { T }<450^{\circ} \mathrm{C} \\
\text { Specific sample } \\
\text { thermocouples, LVDT, } \\
\text { in-situ loading }\end{array}$ & $\begin{array}{l}\text { HT MICA } \\
\text { Static inert gas } \\
\text { T }>1000^{\circ} \mathrm{C} \\
\text { Gen. IV samples } \\
\text { Instrumentation: } \\
\text { to be discussed }\end{array}$ \\
\hline
\end{tabular}




\section{ceaden MICA test device: "Instrumented" configuration}

MICA : Material Irradiation CApsule

$\checkmark$ Based on OSIRIS technologies: CHOUCA / PHAETON

$\Rightarrow$ at least, the same performances

$\checkmark$ Available at JHR start-up

$\checkmark$ Experimental volume:

$\phi 24 \mathrm{~mm} \times 600 \mathrm{~mm}$

Upper volume (instrumentation):

$\phi 24 \mathrm{~mm} \times 2400 \mathrm{~mm}$ (max.)

Lower volume (instrumentation):

$\phi 24 \mathrm{~mm} \times 700 \mathrm{~mm}$ (max)

\begin{tabular}{|c|c|}
\hline $\begin{array}{l}\text { "Standard" MICA } \\
\text { Static NaK coolant } \\
\text { T }<450^{\circ} \mathrm{C} \\
\text { Many samples } \\
\text { Simplified instrumentation: } \\
\text { thermocouples }\end{array}$ & $\begin{array}{l}\text { "Instrumented" MICA } \\
\text { Static NaK coolant } \\
\text { T }<450^{\circ} \mathrm{C} \\
\text { Specific sample } \\
\text { * Evolved instrumentation: } \\
\text { thermocouples, LVDT, } \\
\text { in-situ loading }\end{array}$ \\
\hline
\end{tabular}

\section{MELODIE experiment}

$\checkmark$ Cladding sample

$\checkmark$ In-situ loading: biaxial stress

(internal pressure + tensile/compressive load)

Scanning diameter gauge and elongation during irradiation
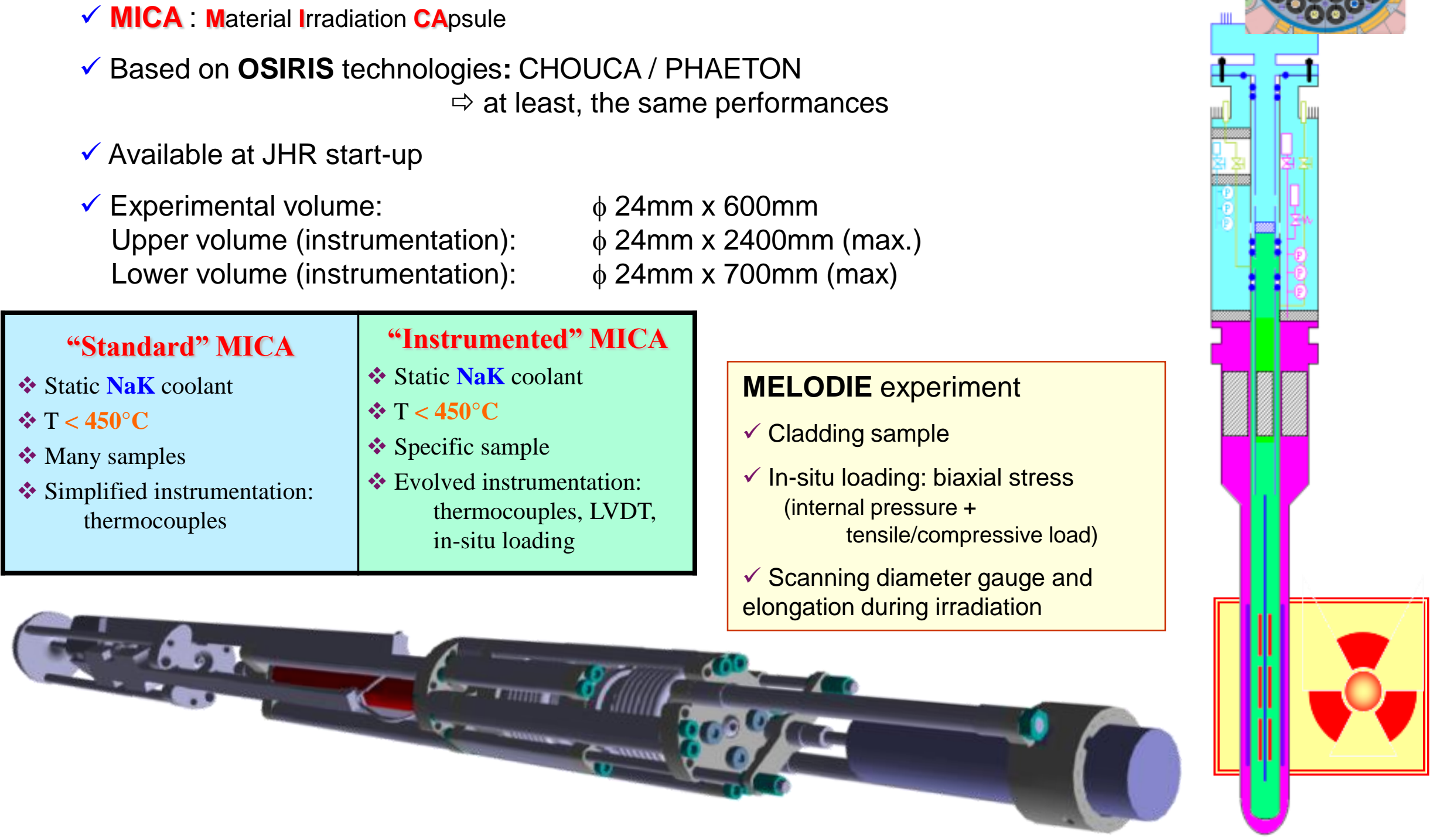

$2^{\text {nd }}$ International Workshop MINOS, November 4-6 2015, Cadarache | PAGE 23 


\section{ceaden MICA test device: "Instrumented" configuration}

$\checkmark$ MICA : Material Irradiation CApsule

$\checkmark$ MELODIE experiment: ..... To prepare MICA instrumented rigs
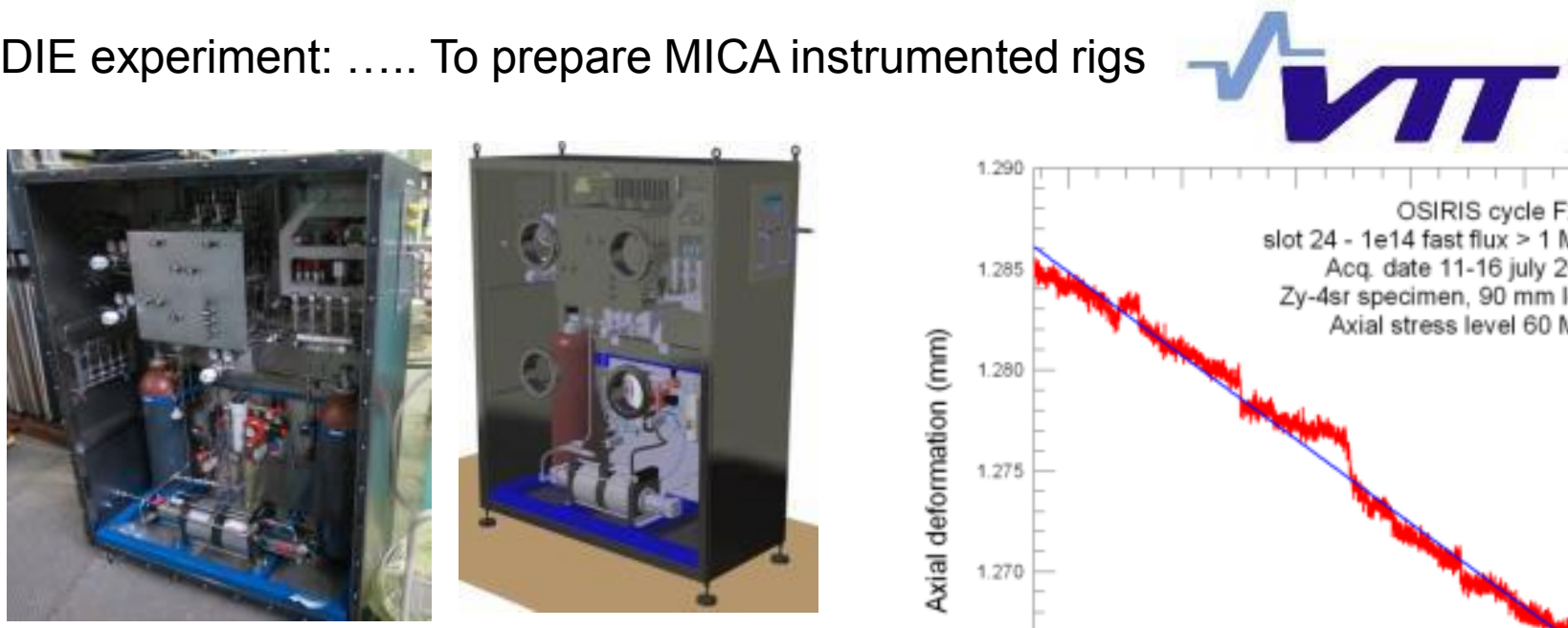

Successful tests of the online measurement of axial deformations under stress

Prototype exhibited already the breakthrough

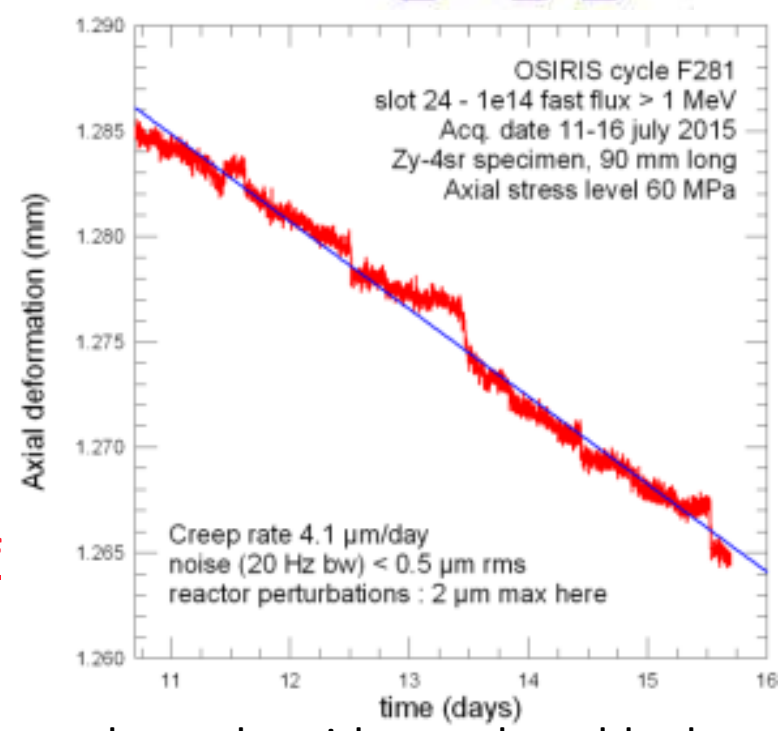
capability to measure a creep rate in a week (several months with " cook-and-look » irradiation devices)

Successful tests of the offline measurement of diametral deformations

Next step: early 2016 , feedback from this first irradiation campaign, optimise the device (MELODIE2)

CEA-VTT investigation for possible irradiation capacity within European Partners to finalise qualification and prepare the Industrial device for JHR

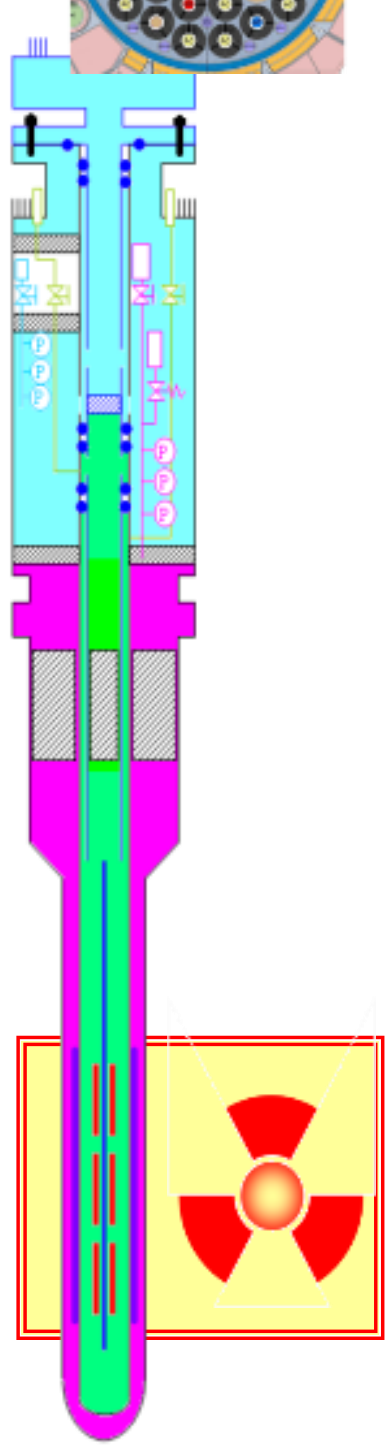




\section{ceaden CALIPSO test device}

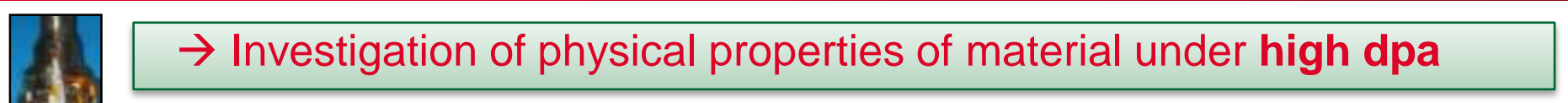

- Thermodynamic loop integrated within the test device

$>$ Heat Exchanger (HE) / Electrical Heater (EH)

$<$ > Innovative electromagnetic pump (L $450 \mathrm{~mm}, \mathrm{D} 80 \mathrm{~mm}) \rightarrow$ NaK flow $\left(2 \mathrm{~m}^{3} / \mathrm{h}\right)$

- Improvement of the sample temperature mastering

$>$ From 250 up to $450^{\circ} \mathrm{C}$ (setting of $\mathrm{HE} \& \mathrm{EH}$ parameters)

$>\Delta \theta<8^{\circ} \mathrm{C}(\mathrm{Tmax}-\mathrm{Tmin}$ all along the samples stack $)$

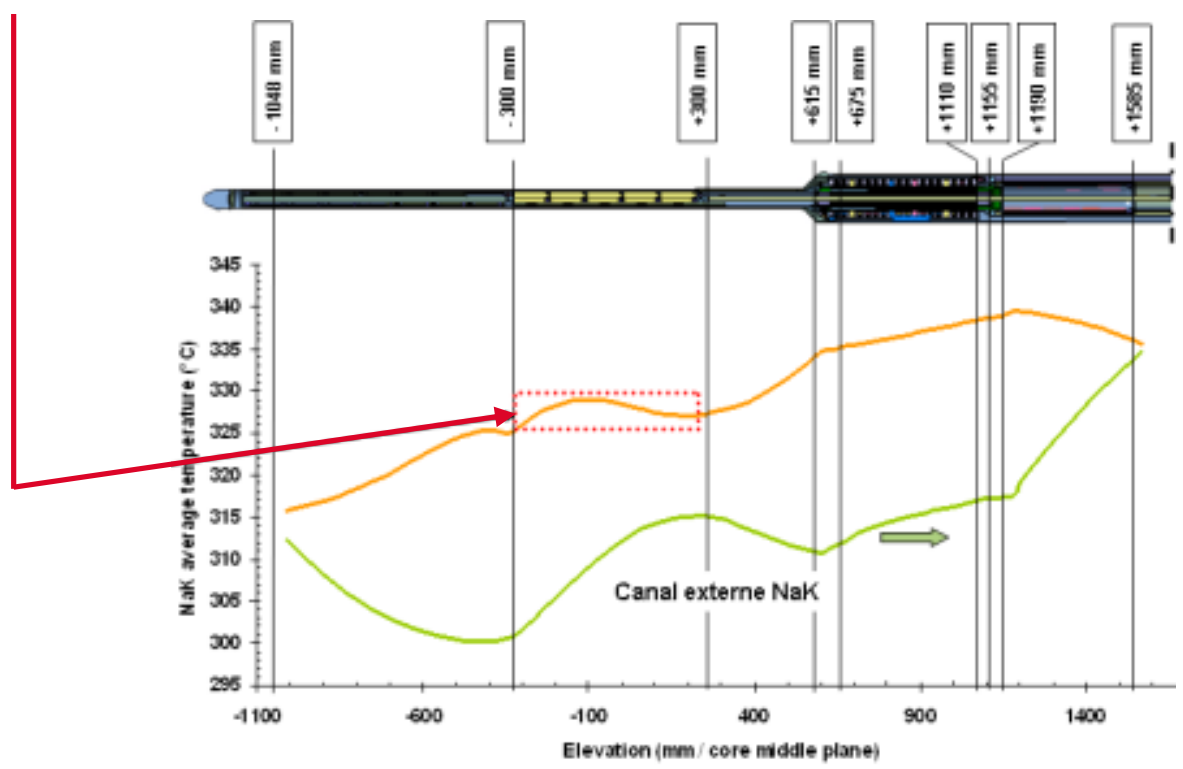

- On-going qualification of the design with a CALIPSO prototype

$>$ First successful tests of the electromagnetic pump

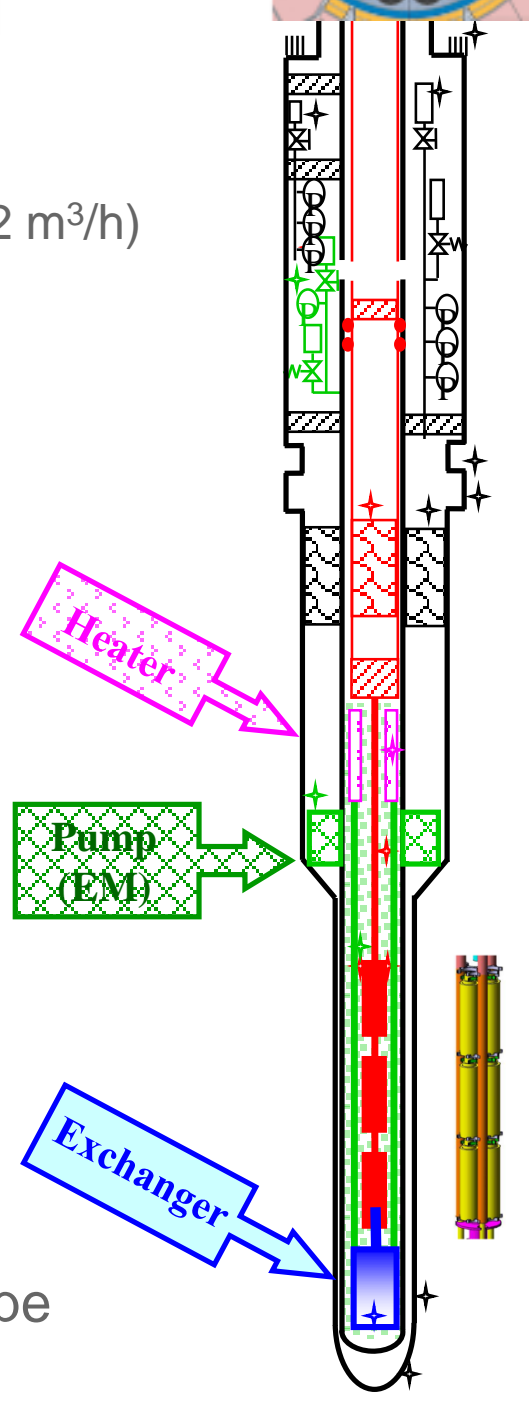

$2^{\text {nd }}$ International Workshop MINOS, November 4-6 2015, Cadarache | PAGE 25 


\section{ceaden OCCITANE test device}

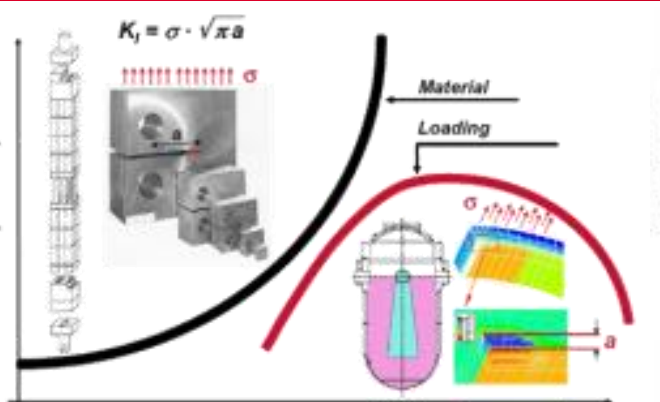

Tempenture

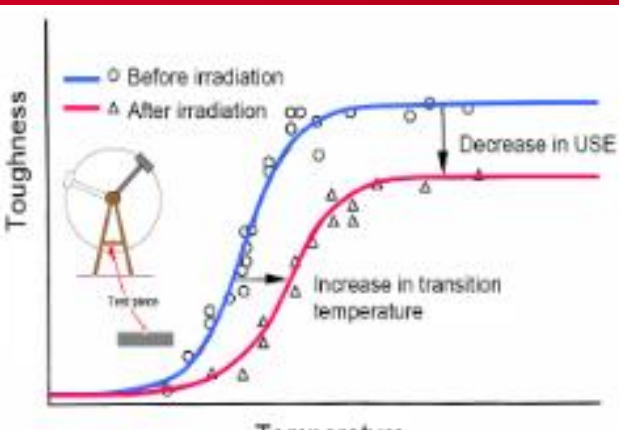

Temperature

OCCITANE : Out-of-Core Capsule for Irradiation Testing of Ageing by Neutrons

- Static Helium capsule

$>$ Based on the OSIRIS feedback (IRMA test device, 150 irradiation cycles)

- Ex-core location

$>$ Fixed location

> Dose: up to $100 \mathrm{mdpa} / \mathrm{y}(1 \mathrm{MeV})$

$>$ Neutron shields

- Samples temperature adjustment

> $230-300^{\circ} \mathrm{C}$

$>$ Gamma heating

$>$ Gas gap dimension

$>$ Electric heating elements

- At least, 18 thermocouples, and 45 dose integrators

$\checkmark$ Equivalent carrying volume: $30 \times 62.5 \times 500 \mathrm{~mm}^{3}$

$\checkmark$ Helium gas

$\checkmark 230-300^{\circ} \mathrm{C}$ (furnace with 6 heating zones)

$\checkmark 100 \mathrm{mdpa} /$ year

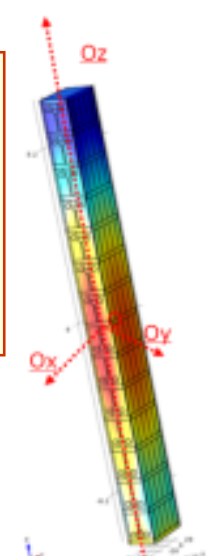

i
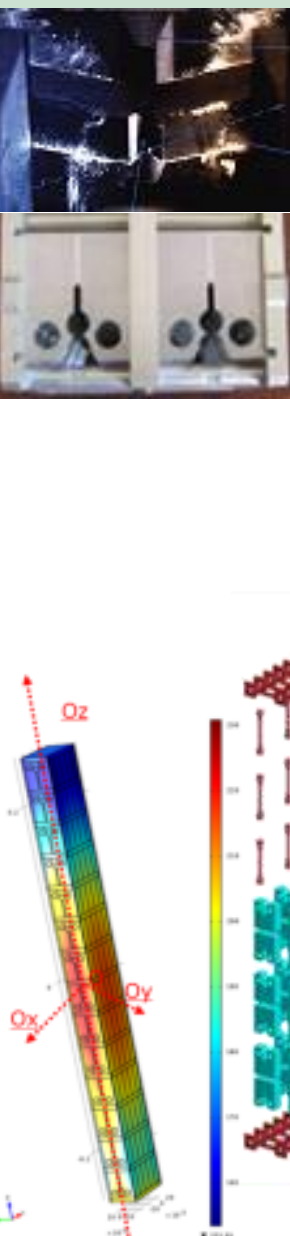


\section{ceaden CLOE test device}
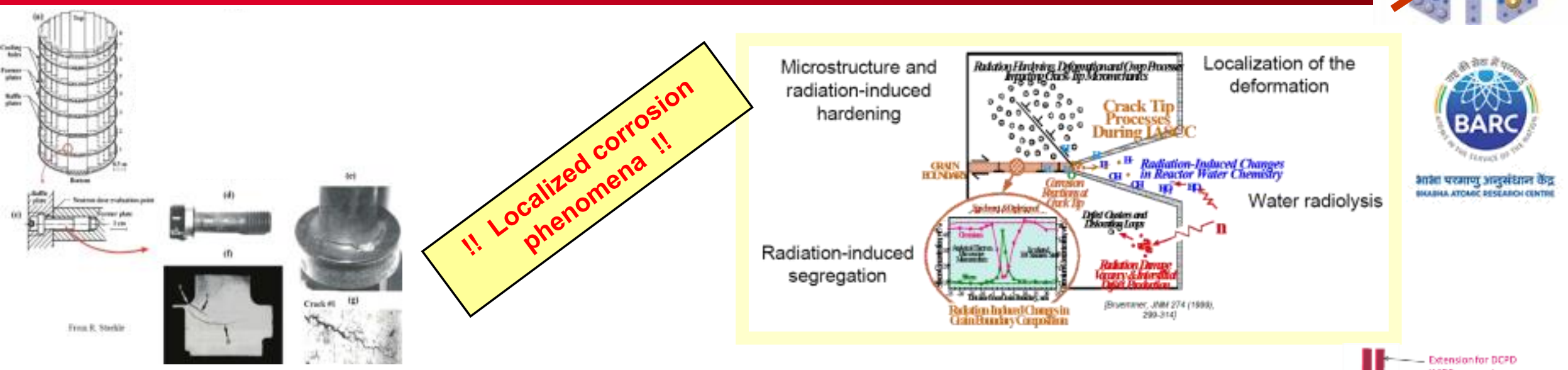

$\Leftrightarrow$ Need of a corrosion loop to perform integral experiments

- India in-kind contribution (DAE-BARC)

- CEA corrosion loops feedback, MTR+i3 European project

- LWR conditions: well controlled and adjusted water chemistry, temperatures, ...

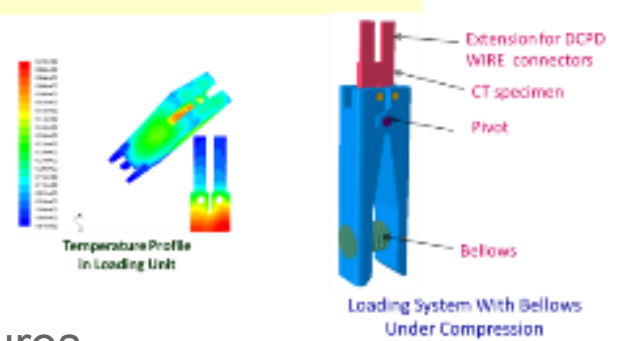

- Fixed location

Ex-core with a large diameter

$>$ In-core with a smaller diameter

(taking into account safety aspect)

- In-situ measurements: ECP, $\mathrm{pH}, \mathrm{H} 2$,

load, LVDT, cracking propagation, DCPD

$\stackrel{\leftrightarrow}{\hookrightarrow}$ Corrosion loop for LWR conditions

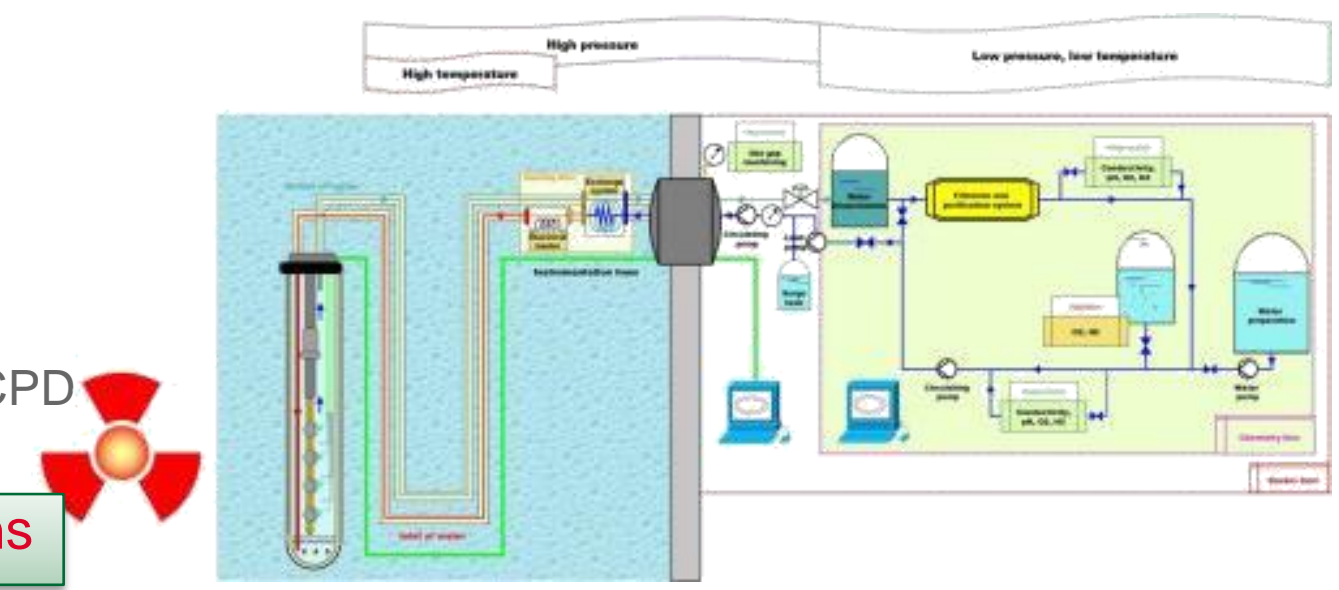




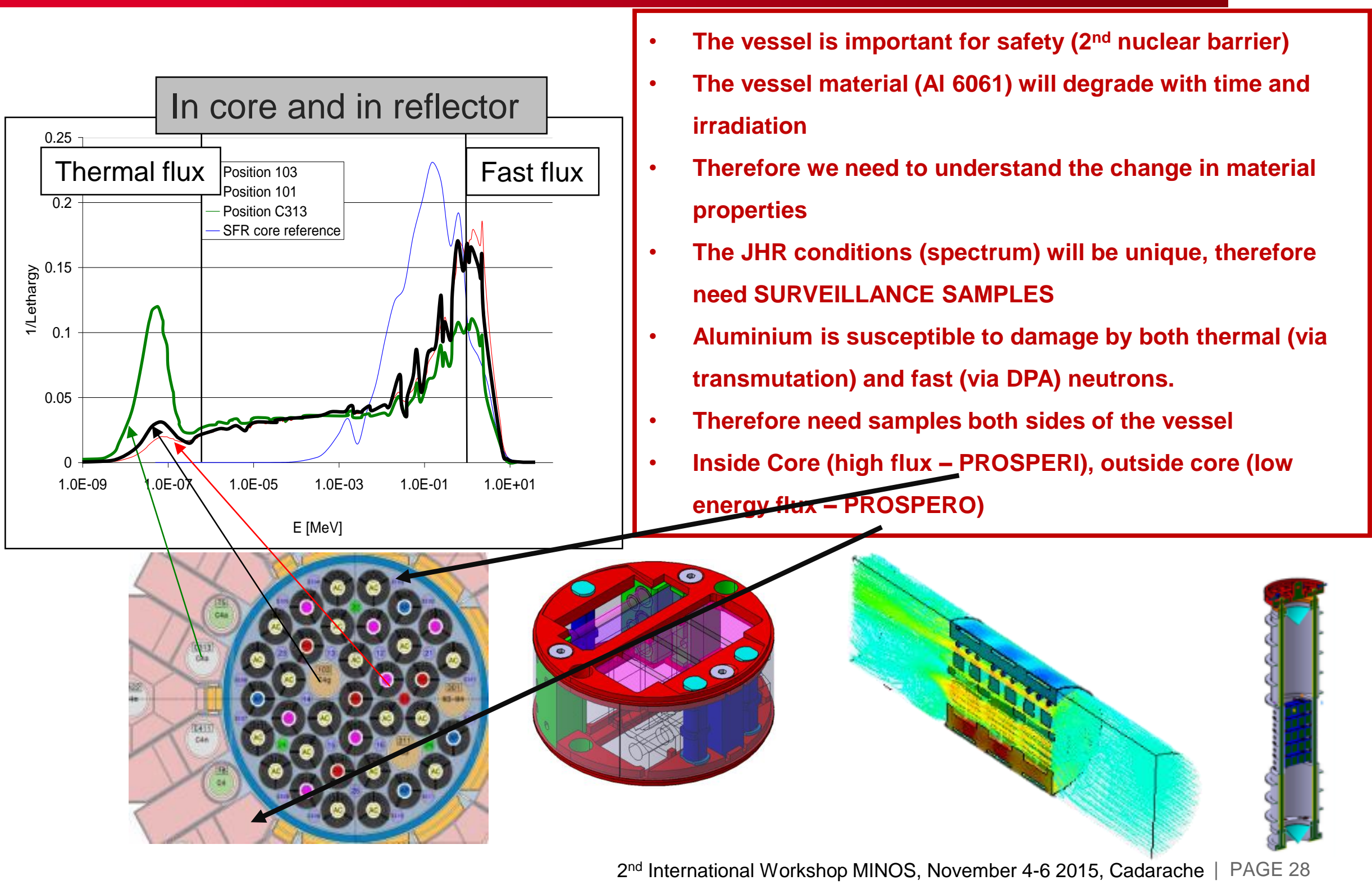



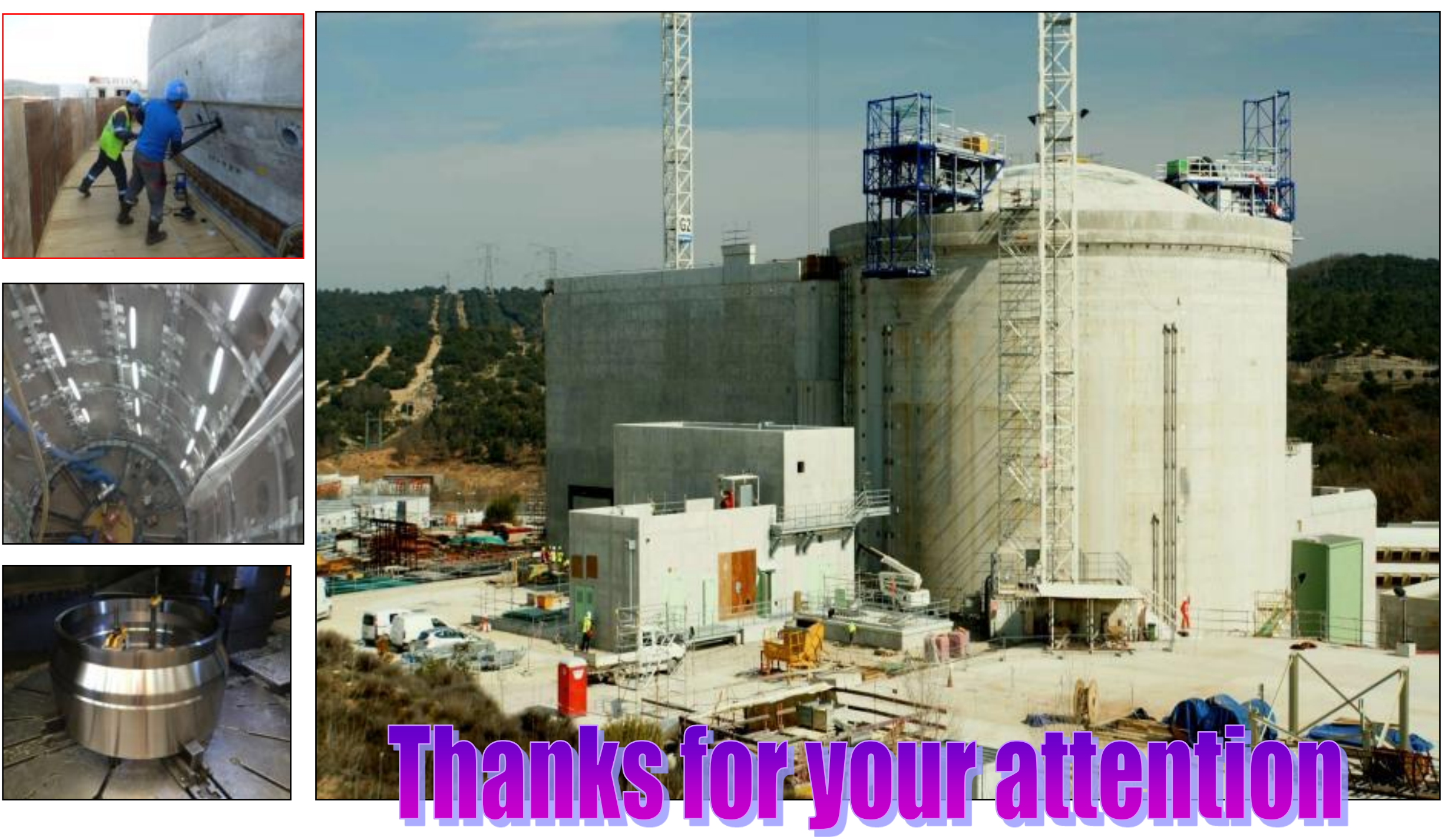

$2^{\text {nd }}$ International Workshop MINOS, November 4-6 2015, Cadarache | PAGE 29 


\section{ceaden JHR facility \& experimental capacity: General architecture}

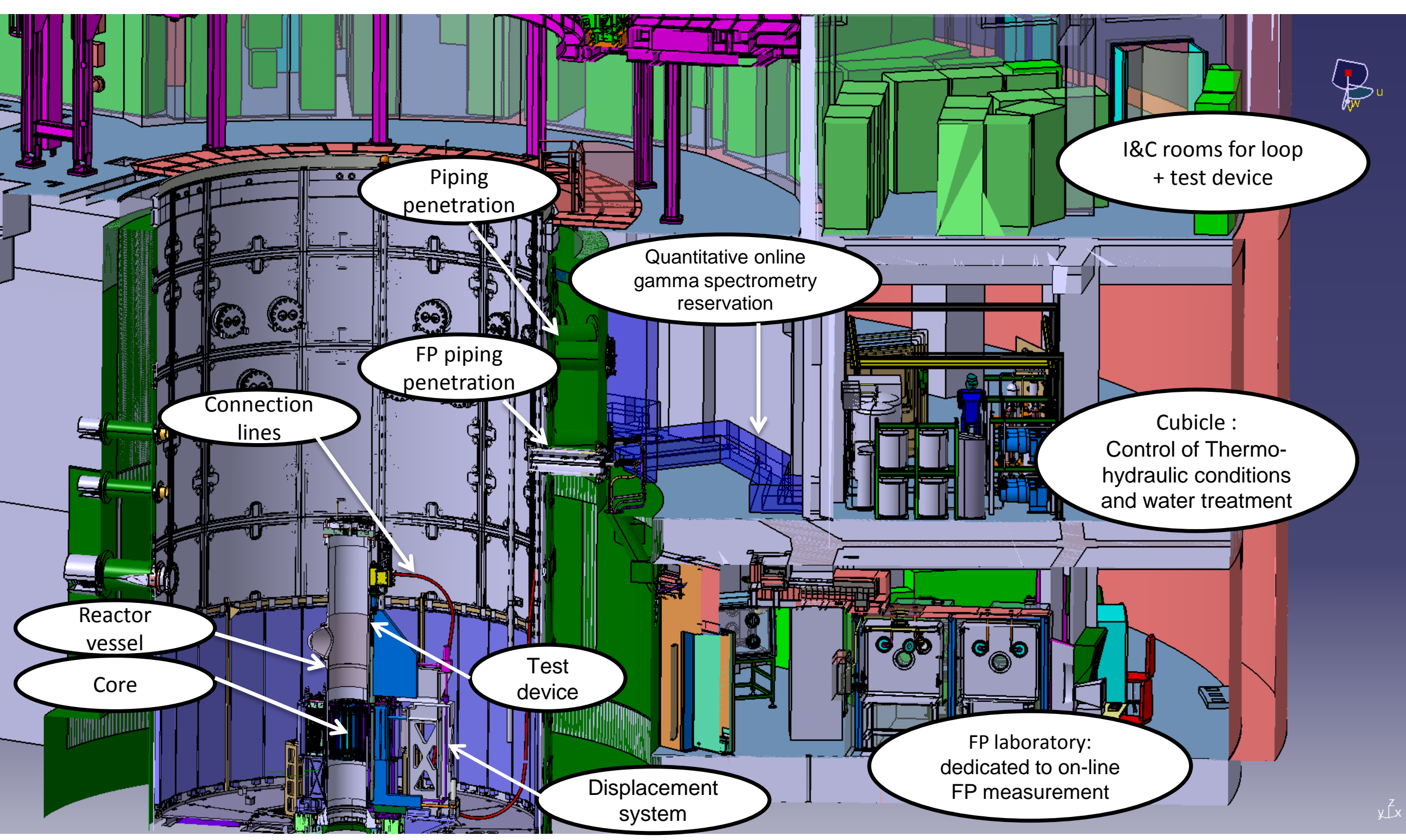




\section{Material and fuel irradiation needs in the nuclear industry}

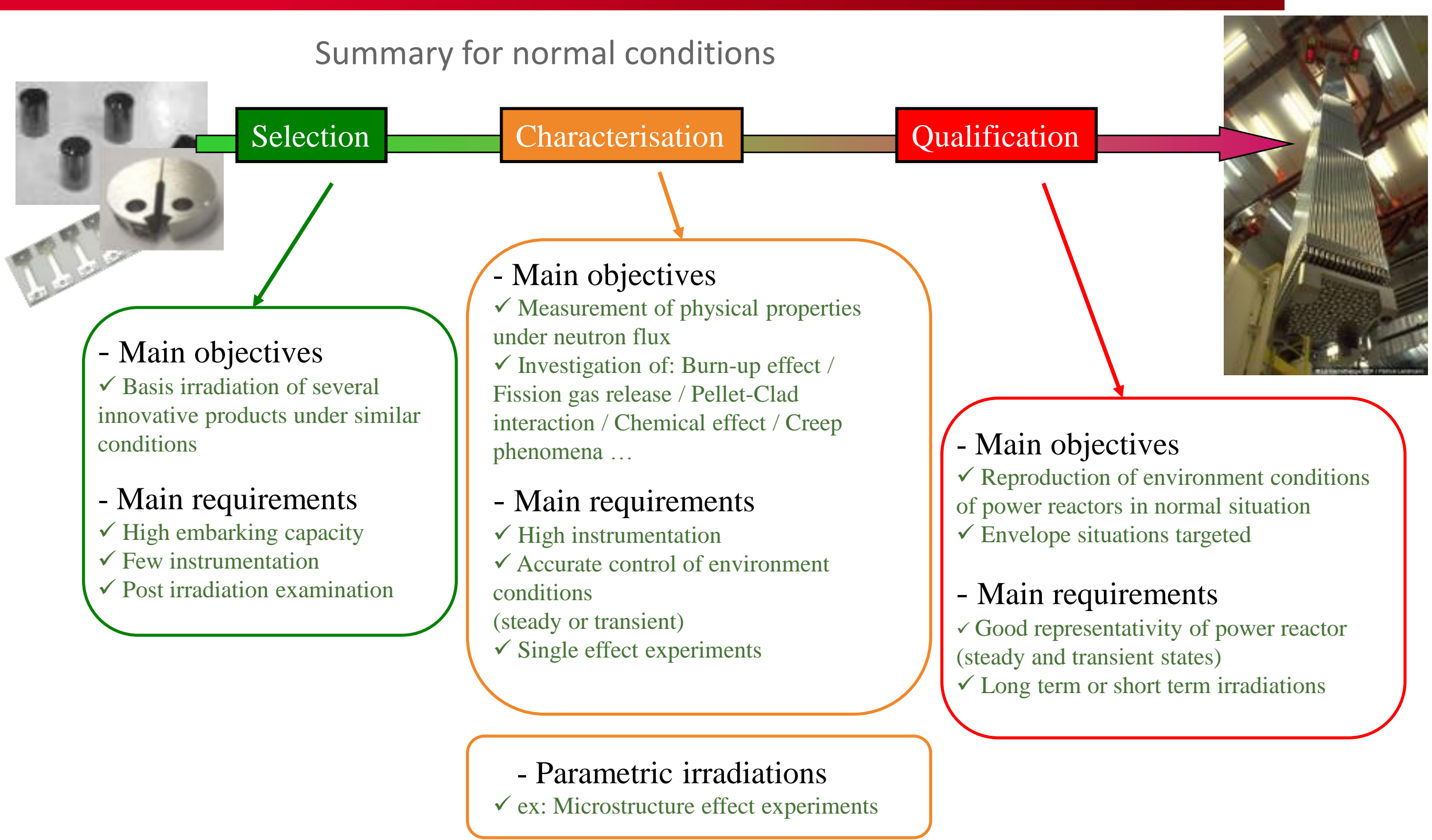




\section{ceaden MICA test device: "Instrumented" configuration}

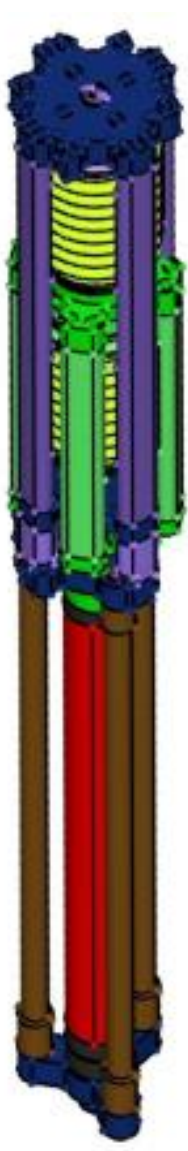

- Technical goals

- $\quad$ Study of LWR cladding irradiation creep

- Real time control of the biaxial stress

+ online measurement of the biaxial creep

- OSIRIS environment

- Sample holder in a CHOUCA capsule $\rightarrow$ Similar to MICA capsule

- $\quad 350^{\circ} \mathrm{C}$, static NaK coolant $\rightarrow$ Similar to MICA capsule

E Biaxial stress controlled in real time

- $\quad$ Specimen pressurization $\rightarrow$ Max pressure 160 bar

- $\quad$ Push-pull axial loading unit

- Hoop Stress limit: $\sigma_{\Theta}=120 \mathrm{MPa}$, Axial stress limit: $\sigma_{\mathrm{z}}=180 \mathrm{MPa}$

- Online biaxial measurement of creep strain IFe

- Continuous measurement of axial strain with a 5-wire LVDT

- Periodical measurement of hoop strain with a diameter gauge (DG)
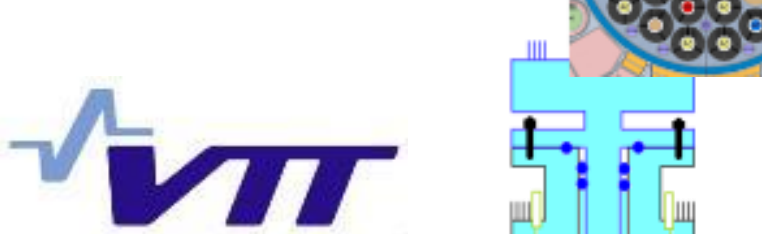

000

MICA : Material Irradiation CApsule

.... To prepare MICA instrumented rigs

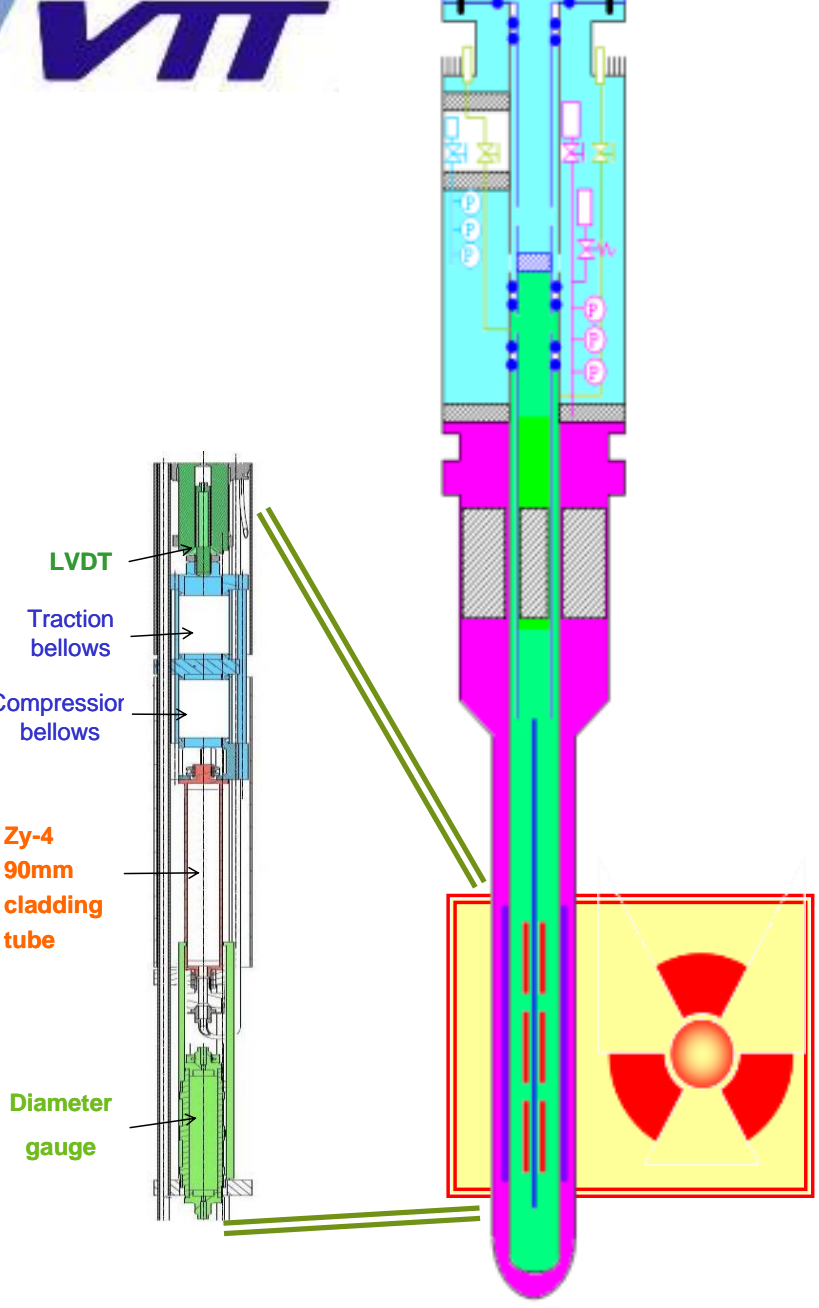




\section{ceaden MICA test device: "Instrumented" configuration}

$\checkmark$ MICA : Material Irradiation CApsule

$\checkmark$ MELODIE experiment:

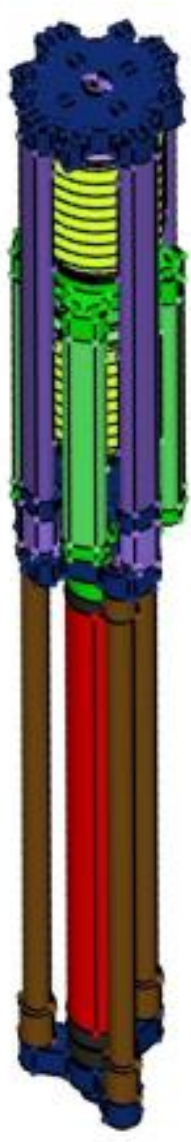

LVDT-80 For DG mover
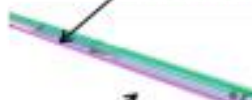

DG mover

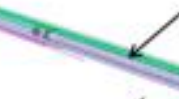

for moving cables
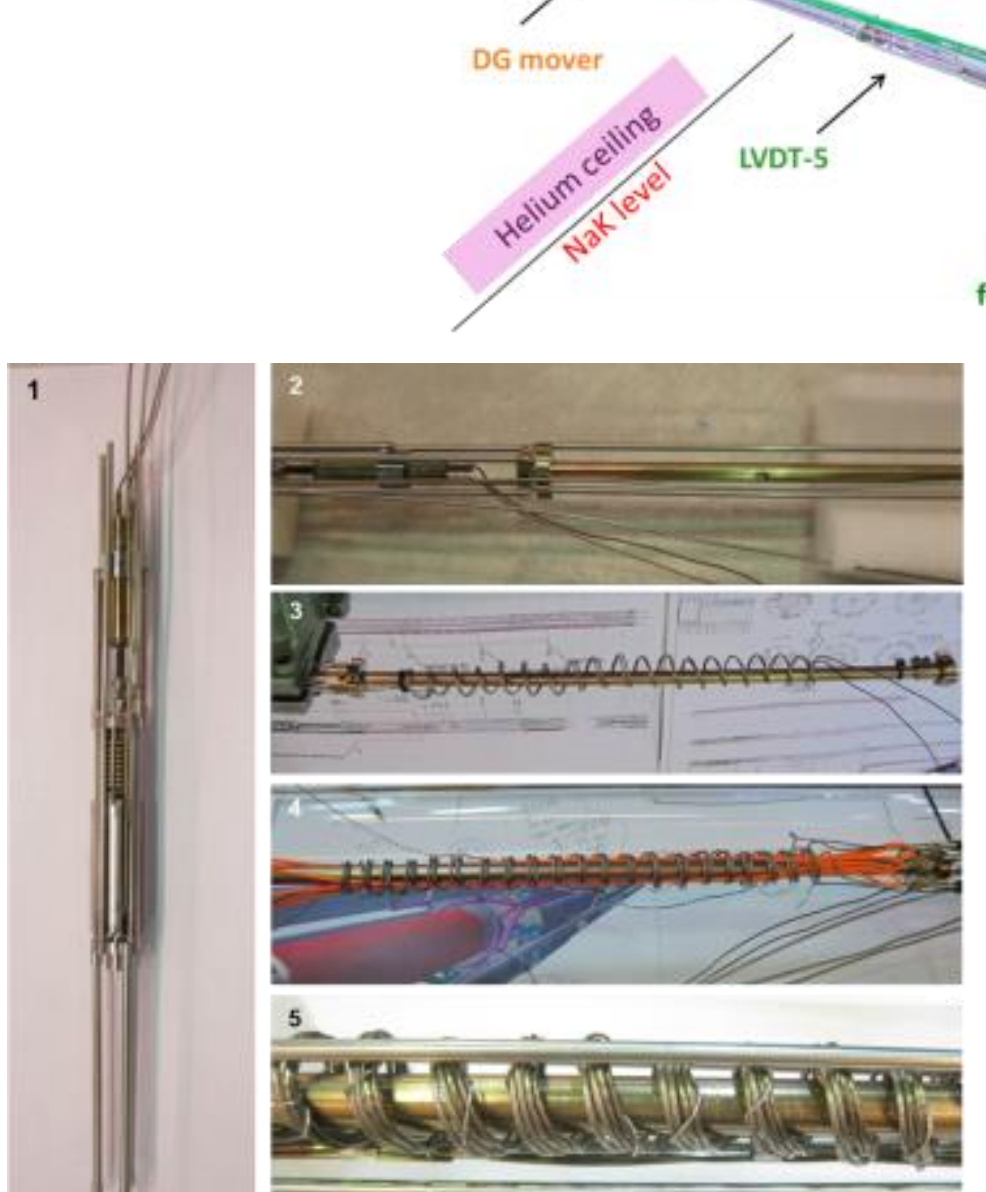

DG feelers
Push-pull bellows

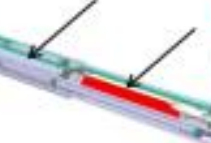

cladding tube

(5 TC en z et $\theta$ )

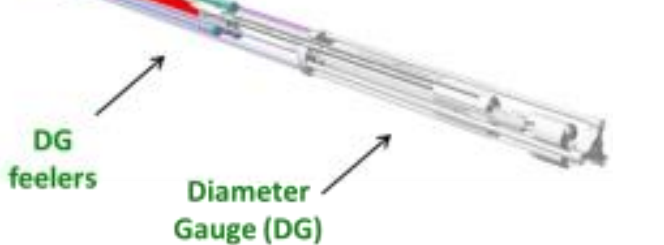

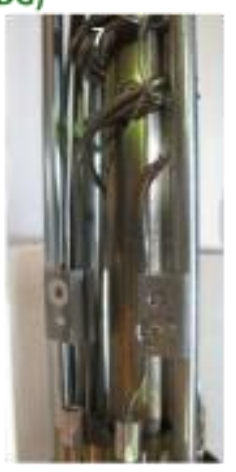

1. Loading module

2. Connecting loading module and lower rig tube

3. Winding of two DG cables

4. Winding of TC7 and TC8 cables

5. Braiding of cables together with SS wire

6. Locking cables to the rig tube

7. Locking cables to the mover posts 1

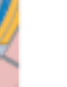




\section{ceaden MICA test device: "Instrumented" configuration}

$\checkmark$ MICA : Material Irradiation CApsule

$\checkmark$ MELODIE experiment: ..... To prepare MICA instrumented rigs

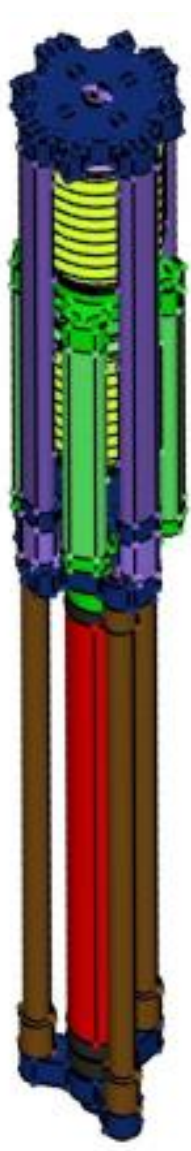

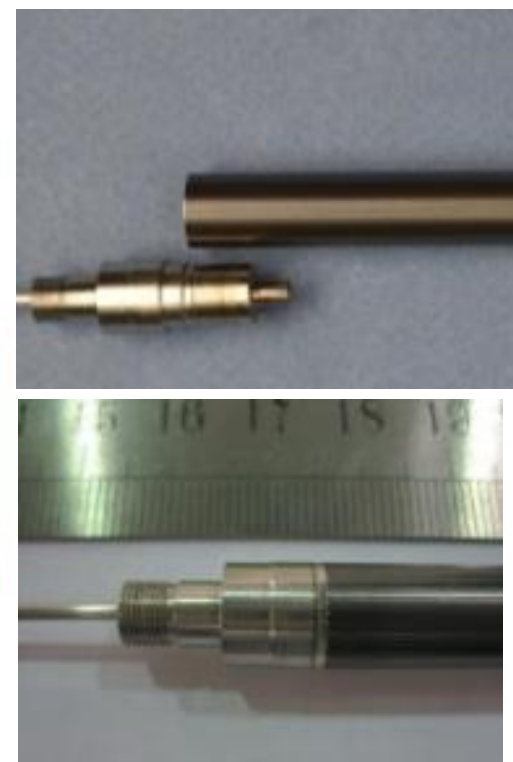

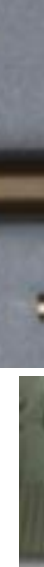

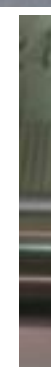
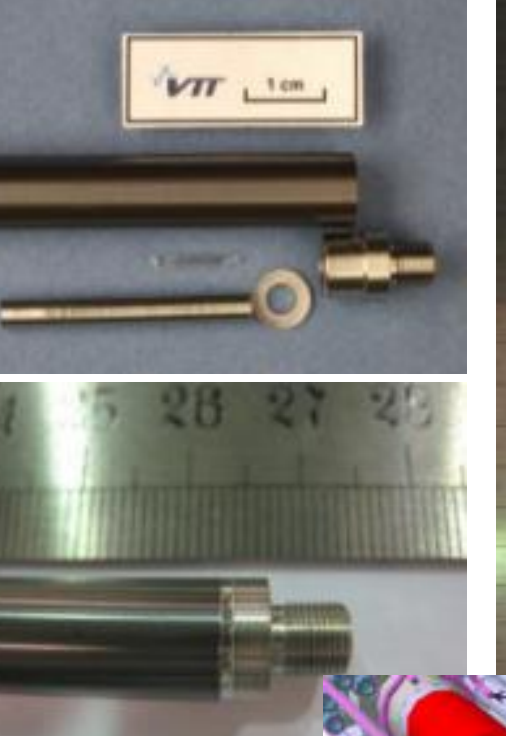

רT
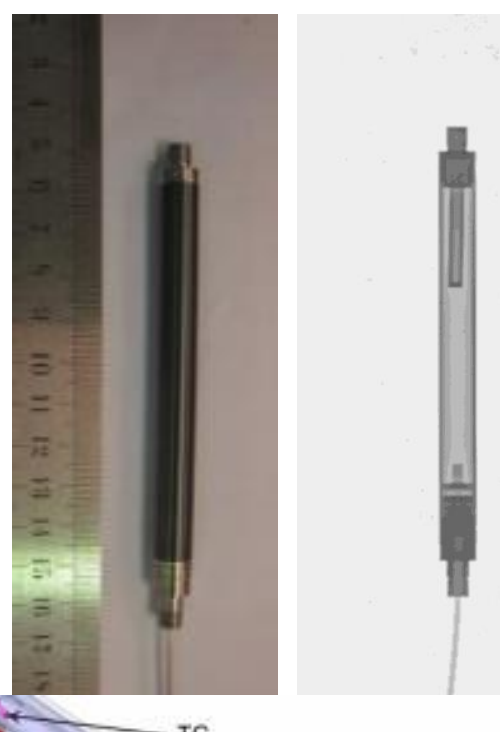
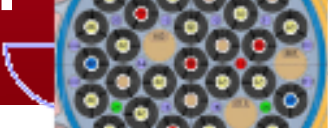

$\because \% 0$
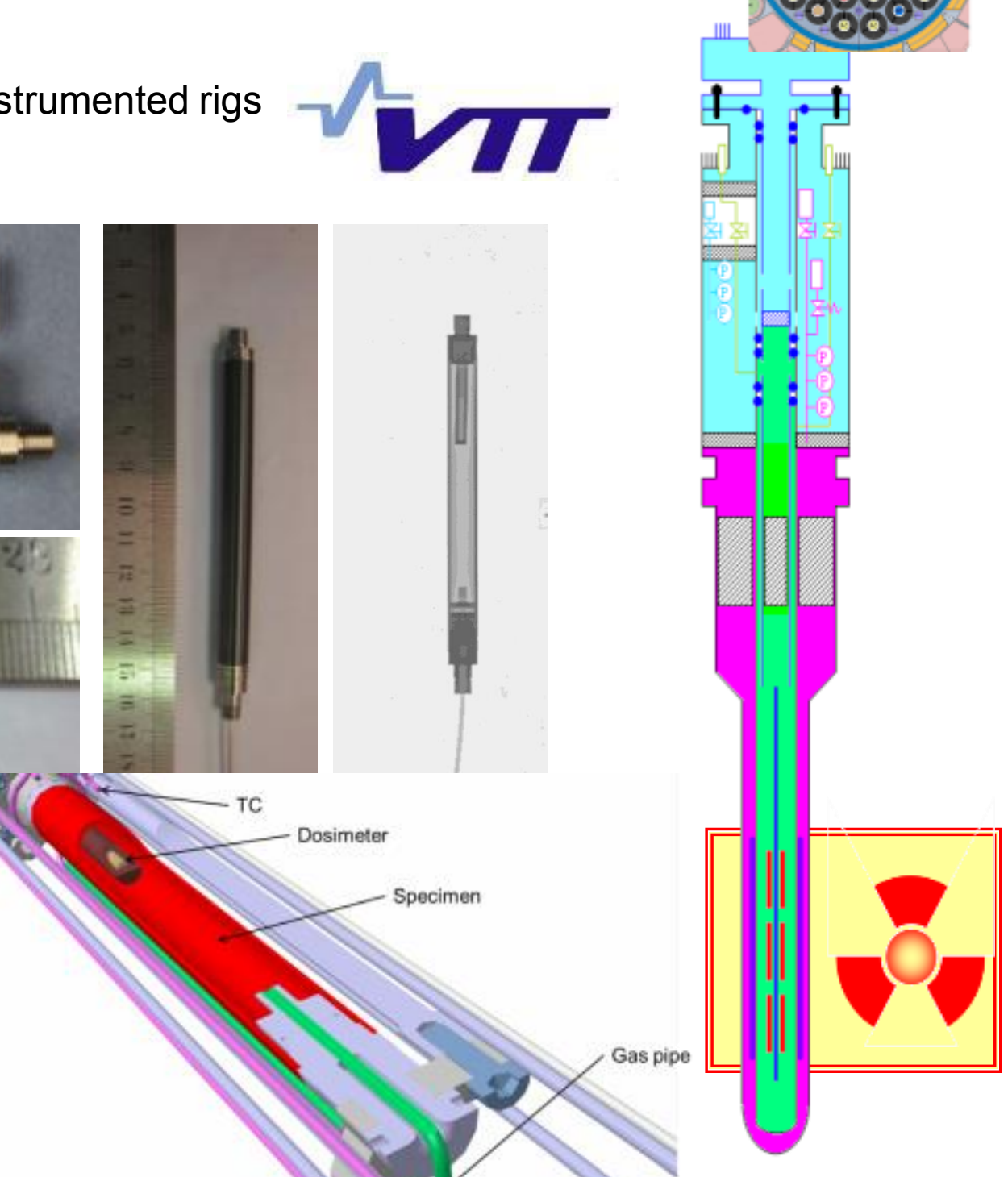

Specimen

Gas pipe

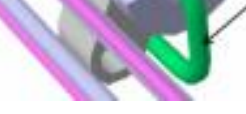




\section{ceaden CALIPSO test device}

\section{SOPRANO Facility :}

$\rightarrow$ Performed first qualification tests with a CALIPSO prototype in 2014

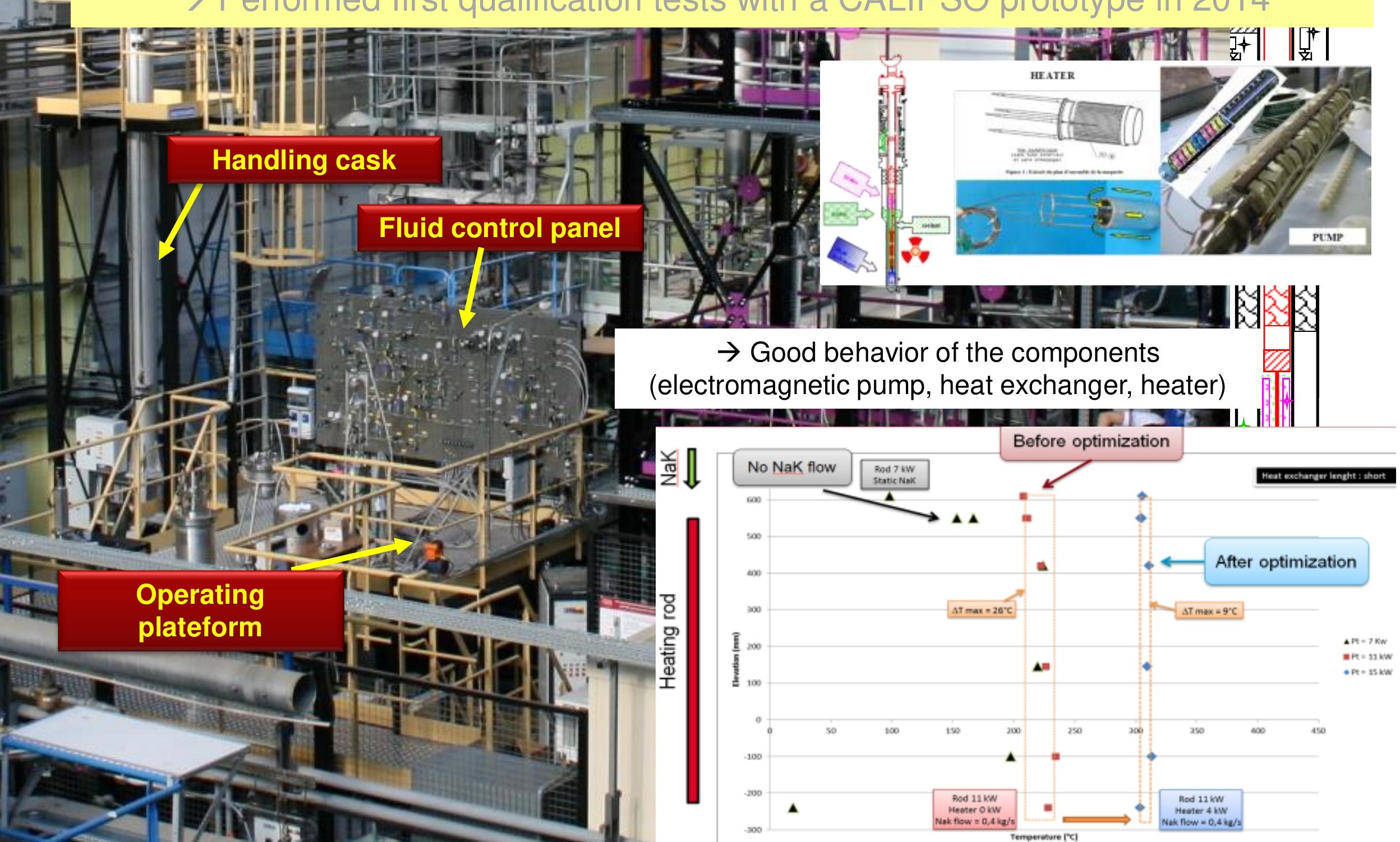


Sample examination in hot cells I

Gamma and X-Ray tomography systems

Multipurpose test benches

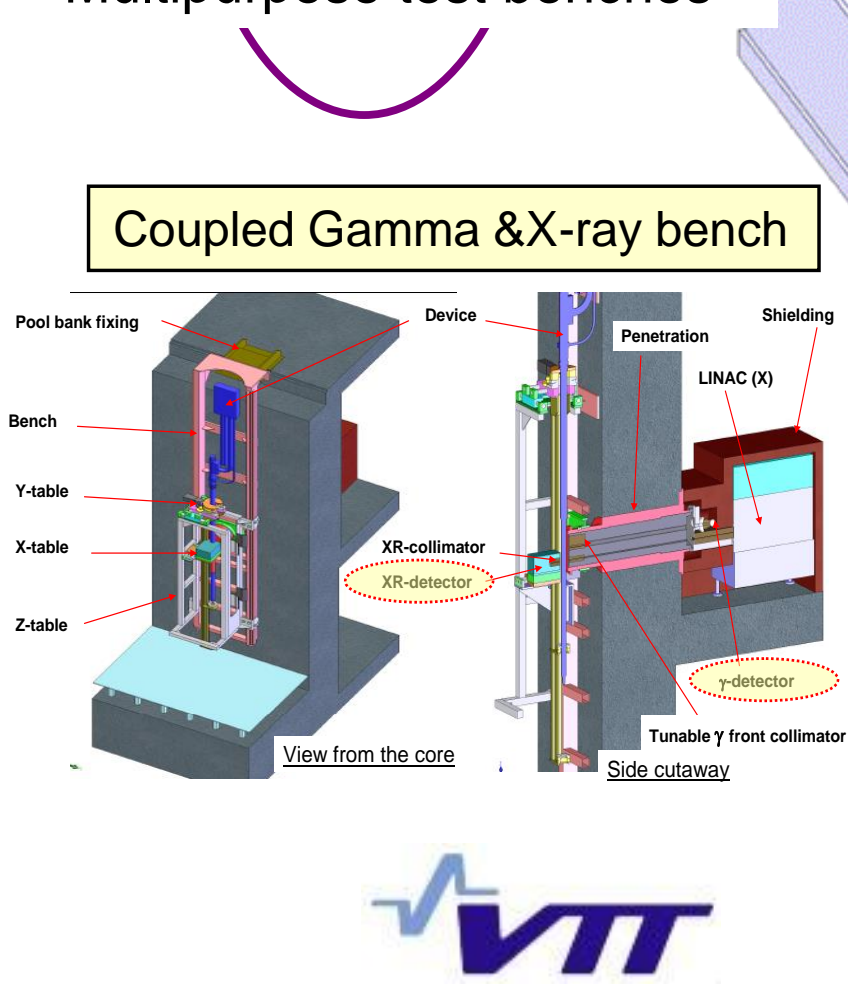

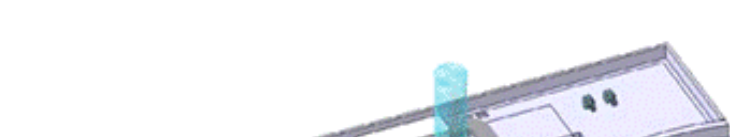
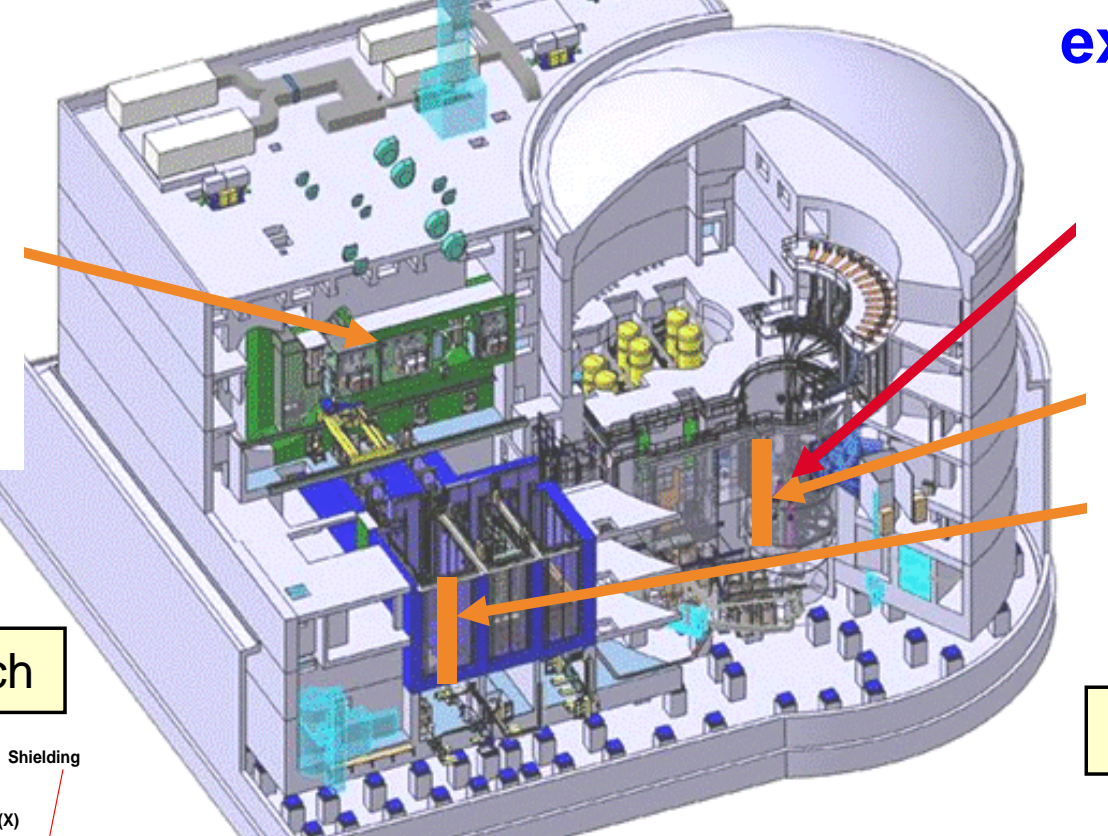

Initial checks of the experimental loading Adjustment of the experimental protocol On-site NDE tests after the irradiation phase
Test device examination in pools Neutron imaging system in reactor pool

Coupled X-ray \& $\gamma$ bench in reactor pool

Coupled X-ray \& $\gamma$ bench in storage pool

Neutron Imaging System 
